(c) 2005 International Press

Adv. Theor. Math. Phys. 9 (2005) 603-665

\title{
Topological M-theory
}

\section{as unification of form theories}

\section{of gravity}

\section{Robbert Dijkgraaf, ${ }^{1}$ Sergei Gukov, ${ }^{2}$ Andrew Neitzke, ${ }^{2}$ Cumrun Vafa ${ }^{2}$}

\author{
${ }^{1}$ Institute for Theoretical Physics \& \\ Korteweg-de Vries Institute for Mathematics, \\ University of Amsterdam, \\ 1018 XE Amsterdam, The Netherlands \\ ${ }^{2}$ Jefferson Physical Laboratory, Harvard University, \\ Cambridge, MA 02138, USA
}

\begin{abstract}
We introduce a notion of topological M-theory and argue that it provides a unification of form theories of gravity in various dimensions. Its classical solutions involve $G_{2}$ holonomy metrics on 7 -manifolds, obtained from a topological action for a 3-form gauge field introduced by Hitchin. We show that by reductions of this 7-dimensional theory, one can classically obtain 6-dimensional topological A and B models, the self-dual sector of loop quantum gravity in four dimensions, and Chern-Simons gravity in 3 dimensions. We also find that the 7 dimensional M-theory perspective sheds some light on the fact that
\end{abstract}

e-print archive: http://lanl.arXiv.org/abs/hep-th/0411073 
the topological string partition function is a wavefunction, as well as on S-duality between the A and B models. The degrees of freedom of the A and $\mathrm{B}$ models appear as conjugate variables in the 7-dimensional theory. Finally, from the topological M-theory perspective, we find hints of an intriguing holographic link between non-supersymmetric Yang-Mills in four dimensions and A model topological strings on twistor space.

\section{Introduction}

The search for a quantum theory of gravity has been a source of puzzles and inspirations for theoretical physics over the past few decades. The most successful approach to date is string theory; but, beautiful as it is, string theory has many extra aspects to it which were not asked for. These include the appearance of extra dimensions and the existence of an infinite tower of increasingly massive particles. These unexpected features have been, at least in some cases, a blessing in disguise; for example, the extra dimensions turned out to be a natural place to hide the microstates of black holes, and the infinite tower of particles was necessary in order for the AdS/CFT duality to make sense. Nevertheless, it is natural to ask whether there could be simpler theories of quantum gravity. If they exist, it might be possible to understand them more deeply, leading us to a better understanding of what it means to quantize gravity; furthermore, simple theories of gravity might end up being the backbone of the more complicated realistic theories of quantum gravity.

In the past decade, some realizations of this notion of a "simpler" theory of gravity have begun to emerge from a number of different directions. The common thread in all these descriptions is that, in the theories of gravity which appear, the metric is not one of the fundamental variables. Rather, these theories describe dynamics of gauge fields or higher $p$-forms, in terms of which the metric can be reconstructed. These theories generally have only a finite number of fields; we shall call them form theories of gravity.

Notable examples of form theories of gravity are ${ }^{1}$ the description of 3dimensional gravity in terms of Chern-Simons theory, the description of 4dimensional gravity in terms of $S U(2)$ gauge theory coupled to other fields, the description of the target space theory of A model topological strings in

\footnotetext{
${ }^{1}$ One could also include in this list, as will be discussed later in this paper, the case of 2-dimensional gravity in the target space of the non-critical $c=1$ string; in that case, one gets a theory involving a symplectic form on a 2-dimensional phase space, defining a Fermi surface, in term of which the metric and other fields can be reconstructed.
} 
terms of variations of the Kähler 2-form, and the description of the target space theory of the B model in terms of variations of the holomorphic 3 -form.

Meanwhile, recent developments in the study of the topological A and $B$ models suggest that we need a deeper understanding of these theories. On the one hand, they have been conjectured to be S-dual to one another $[1,2]$. On the other hand, the $\mathrm{A}$ model has been related to a quantum gravitational foam $[3,4]$. Moreover, their non-perturbative definition has begun to emerge through their deep connection with the counting of BPS black hole states in four dimensions $[5,6]$. There is also a somewhat older fact still in need of a satisfactory explanation: it has been known for a while that the holomorphic anomaly of topological strings [7] can be viewed as the statement that the partition function of topological string is a state in some 7-dimensional theory, with the Calabi-Yau 3-fold realized as the boundary of space [8] (see also [9]).

Parallel to the new discoveries about topological strings was the discovery of new actions for which the field space consists of "stable forms" [10]. The critical points of these actions can be used to construct special holonomy metrics. A particularly interesting example is a 3-form theory which constructs $G_{2}$ holonomy metrics in seven dimensions. Interestingly enough, as we will explain, the Hamiltonian quantization of this theory looks a lot like a combination of the $\mathrm{A}$ and $\mathrm{B}$ model topological strings, which appear in terms of conjugate variables. All this hints at the existence of a "topological M-theory" in seven dimensions, whose effective action leads to $G_{2}$ holonomy metrics and which can reduce to the topological A and B models.

The main aim of this paper is to take the first steps in developing a unified picture of all these form theories of gravity. Our aim is rather modest; we limit ourselves to introducing some of the key ideas and how we think they may be related, without any claim to presenting a complete picture. The 7-dimensional theory will be the unifying principle; it generates the topological string theories as we just noted, and furthermore, the interesting gravitational form theories in 3- and 4-dimensions can be viewed as reductions of this 7-dimensional form theory near associative and coassociative cycles.

We will also find another common theme. The form theories of gravity naturally lead to calibrated geometries, which are the natural setting for the definition of supersymmetric cycles where branes can be wrapped. This link suggests an alternative way to view these form theories, which may indicate how to define them at the quantum level: they can be understood as counting the BPS states of wrapped branes of superstrings. Namely, recall that in the superstring there is an attractor mechanism relating the charges of the 
black hole (the homology class of the cycle they wrap on) to specific moduli of the internal theory (determining the metric of the internal manifold). We will see that the attractor mechanism can be viewed as a special case of the general idea of obtaining metrics from forms.

The organization of this paper is as follows. In Section 2, we provide evidence for the existence of topological M-theory in 7 dimensions. In particular, we use the embedding of the topological string into the superstring to give a working definition of topological M-theory in terms of topological strings in 6 dimensions, with an extra circle bundle providing the " 11 th" direction. We also give a more extensive discussion of how the very existence of topological M-theory could help resolve a number of puzzles for topological strings in 6 dimensions. In Section 3, we give a short review of some form gravity theories in dimensions 2, 3, 4, and 6. In Section 4, we discuss some new action principles constructed by Hitchin, which lead to effective theories of gravity in 6- and 7-dimensions. These gravity theories are related to special holonomy manifolds and depend on the mathematical notion of "stable form," so we begin by reviewing these topics; then we introduce Hitchin's actions in 6 and 7 dimensions, as well as a classical Hamiltonian formulation of the 7-dimensional theory. In Section 5, we argue that these new gravity theories in 6 dimensions are in fact reformulations of the target space dynamics of the $\mathrm{A}$ and $\mathrm{B}$ model topological string theories. In Section 6 , we show how the 7-dimensional theory reduces classically to the three-, four-, and 6-dimensional gravity theories we reviewed in Section 3. In Section 7, we discuss canonical quantization of the 7-dimensional theory; we show that it is related to the A and B model topological strings, and we argue that this perspective could shed light on the topological S-duality conjecture. In Section 8, we reinterpret the gravitational form theories as computing the entropy of BPS black holes. In Section 9, we discuss a curious holographic connection between twistor theory and the topological $G_{2}$ gravity. In Section 10, we discuss possible directions for further development of the ideas discussed in this paper. Finally, in an appendix, we discuss an interesting connection between the phase space of topological M-theory and $\mathcal{N}=1$ supersymmetric vacua in four dimensions.

\section{Evidence for topological M-theory}

In order to define a notion of topological M-theory, we exploit the connection between the physical superstring and the physical M-theory. Recall that we know that topological strings make sense on Calabi-Yau 3-folds, and topological string computations can be embedded into the superstring. It is natural to expect that the dualities of the superstring, which 
found a natural geometric explanation in M-theory, descend to some dualities in topological theories, which might find a similar geometric explanation in topological M-theory. Thus a natural definition of topological M-theory is that it should be a theory with one extra dimension relative to the topological string, for a total of 7 . Moreover, we should expect that $M$-theory on $M \times \mathbf{S}^{1}$ is equivalent to topological strings on $M$, where $M$ is a Calabi-Yau manifold. More precisely, here we are referring to the topological A model on $M$. The worldsheets of A model strings are identified with M-theory membranes which wrap the $\mathbf{S}^{1}$. Later we will see that in some sense the M-theory formalism seems to automatically include the B model along with the A model, with the two topological string theories appearing as conjugate variables. The topological string should be a good guide to the meaning of topological M-theory, at least in the limit where the $\mathbf{S}^{1}$ has small radius. One would expect that the radius of the $\mathbf{S}^{1}$ gets mapped to the coupling constant of the topological string. Of course, topological M-theory should provide an, as yet not well-defined, non-perturbative definition of topological string theory.

So far we only discussed a constant size $\mathbf{S}^{1}$, but we could also consider the situation where the radius is varying, giving a more general 7-manifold. The only natural class of such manifolds which preserves supersymmetry and is purely geometric is the class of $G_{2}$ holonomy spaces; indeed, that there should be a topological theory on $G_{2}$ manifolds was already noted in [11], which studied Euclidean M2-brane instantons wrapping associative 3 -cycles. So consider M-theory on a $G_{2}$ holonomy manifold $X$ with a $U(1)$ action. This is equivalent to the Type IIA superstring, with D6 branes wrapping Lagrangian loci on the base where the circle fibration degenerates. We define topological $M$-theory on $X$ to be equivalent to $A$ model topological strings on $X / U(1)$, with Lagrangian D-branes inserted where the circle fibration degenerates. This way of defining a topological M-theory on $G_{2}$ was suggested in $[12,13]$.

In this setting, the worldsheets of the A model can end on the Lagrangian branes; when lifted up to the full geometry of $X$ these configurations correspond to honest closed 3-cycles which we identify as membrane worldvolumes. Moreover, string worldsheets which happen to wrap holomorphic cycles of the Calabi-Yau lift to membranes wrapping associative 3-cycles of the $G_{2}$ holonomy manifold. So, roughly speaking, we expect that topological M-theory should be classically a theory of $G_{2}$ holonomy metrics, which gets quantum corrected by membranes wrapping associative 3-cycles - in the same sense as the topological A model is classically a theory of Kähler metrics, which gets quantum corrected by strings wrapping holomorphic cycles. We can be a little more precise about the coupling between the membrane 
and the metric: recall that a $G_{2}$ manifold comes equipped with a 3 -form $\Phi$ and a dual 4 -form $G=* \Phi$, in terms of which the metric can be reconstructed. We will see that it is natural to consider this $G$ as a field strength for a gauge potential, writing $G=G_{0}+d \Gamma$; then $\Gamma$ is a 3 -form under which the membrane is charged.

So we have a workable definition of topological M-theory, which makes sense on 7-manifolds with $G_{2}$ holonomy, at least perturbatively to all orders in the radius of the circle. Thus the existence of the theory is established in the special cases where we have a $U(1)$ action on $X$; we conjecture that this can be extended to a theory which makes sense for arbitrary $G_{2}$ holonomy manifolds. This is analogous to what we do in the physical superstring; we do not have an a priori definition of M-theory on general backgrounds, but only in special situations.

Now that we have established the existence of a topological M-theory in seven dimensions (more or less at the same level of rigor as for the usual superstring/M-theory relation), we can turn to the question of what new predictions this theory makes. Indeed, we now suggest that it may solve two puzzles which were previously encountered in the topological string.

There has been a longstanding prediction of the existence of a 7dimensional topological theory from a very different perspective, namely the wavefunction property of the topological string partition function, which we now briefly recall in the context of the $\mathrm{B}$ model. The $\mathrm{B}$ model is a theory of variations $\delta \Omega$ of a holomorphic 3 -form on a Calabi-Yau 3-fold $X$. Its partition function is written $Z_{B}\left(x ; \Omega_{0}\right)$. Here $x$ refers to the zero mode of $\delta \Omega$, $x \in H^{3,0}(X, \mathbb{C}) \oplus H^{2,1}(X, \mathbb{C})$, which is not integrated over in the $\mathrm{B}$ model. The other variable $\Omega_{0}$ labels a point on the moduli space of complex structures on $X$; it specifies the background complex structure about which one perturbs. Studying the dependence of $Z_{B}$ on $\Omega_{0}$, one finds a "holomorphic anomaly equation" [7, 14], which is equivalent to the statement that $Z_{B}$ is a wavefunction [8], defined on the phase space $H^{3}(X, \mathbb{R})$. Namely, different $\Omega_{0}$ just correspond to different polarizations of this phase space, so $Z_{B}\left(x ; \Omega_{0}\right)$ is related to $Z_{B}\left(x ; \Omega_{0}^{\prime}\right)$ by a Fourier-type transform. This wavefunction behavior is mysterious from the point of view of the 6-dimensional theory on $X$. On the other hand, it would be natural in a 7-dimensional theory: namely, if $X$ is realized as the boundary of a 7-manifold $Y$, then path integration over $Y$ gives a wavefunction of the boundary conditions one fixes on $X$.

Another reason to expect a 7-dimensional description comes from the recent conjectures that the $\mathrm{A}$ model and $\mathrm{B}$ model are independent only perturbatively. Namely, each contains non-perturbative objects which could 
naturally couple to the fields of the other. The branes in the A model are wrapped on Lagrangian cycles, the volume of which are measured by some 3 -form, and it is natural to identify this 3-form with the holomorphic 3form $\Omega$ of the B model; conversely, the branes in the B model are wrapped on holomorphic cycles, whose volumes would be naturally measured by the Kähler form $k$ of the A model. This observation has led to the conjecture [1, 2 ] that non-perturbatively both models should include both types of fields and branes, and in fact that the two could even be S-dual to one another. One is thus naturally led to search for a non-perturbative formulation of the topological string which would naturally unify the A and B model branes and fields. Such a unification is natural in the 7-dimensional context: near a boundary with unit normal direction $d t$, the 3 - and 4-forms $\Phi, G$ defining the $G_{2}$ structure naturally combine the fields of the A and B model on the boundary,

$$
\begin{aligned}
& \Phi=\operatorname{Re} \Omega+k \wedge d t, \\
& G=\operatorname{Im} \Omega \wedge d t+\frac{1}{2} k \wedge k .
\end{aligned}
$$

Later we will see that the A and B model fields are canonically conjugate in the Hamiltonian reduction of topological M-theory on $X \times \mathbb{R}$. In particular, the wavefunctions of the A model and the B model cannot be defined simultaneously.

\section{Form theories of gravity in diverse dimensions}

The long-wavelength action of the "topological M-theory" we are proposing will describe metrics equipped with a $G_{2}$ structure. In fact, as we will discuss in detail, the 7-dimensional metric in this theory is reconstructed from the 3 -form $\Phi$ (or equivalently, from the 4 -form $G=* \Phi$ ). This might at first seem exotic: the metric is not a fundamental field of this theory, but rather can be reconstructed from $\Phi$. However, similar constructions have appeared in lower dimensions, where it is believed at least in some cases that the reformulation in terms of forms ("form theory of gravity") is a better starting point for quantization: we know how to deal with gauge theories, and perhaps more general form theories, better than we know how to deal with gravity. Of course, rewriting a classical theory of gravity in terms of classical forms is no guarantee that the corresponding quantum theory exists. We are certainly not claiming that arbitrary form theories make sense at the quantum level! 
Nevertheless, in low dimensions some special form gravity theories have been discussed in the literature, which we believe do exist in the quantum world - and moreover, as we will see, these theories are connected to topological M-theory, which we have already argued should exist.

In this section, we review the form gravity theories in question. They describe various geometries in 2, 3, 4, and 6 dimensions. Here we will discuss mainly their classical description. It is more or less established that the theories discussed below in dimensions 2, 3, and 6 exist as quantum theories, at least perturbatively. In dimensions two and six, this is guaranteed by topological string constructions. In dimension 3 also, the quantum theory should exist since it is known to lead to well defined invariants of 3-manifolds. The 4-dimensional theory, which gives self-dual gravity, is not known to exist in full generality, although for zero cosmological constant, it is related to the Euclidean $\mathcal{N}=2$ string, which is known to exist perturbatively [15]. For the case of non-zero cosmological constant, we will give further evidence that the theory exists at the quantum level by relating it to topological M-theory later in this paper.

\subsection{D form gravity}

By the $2 D$ form theory of gravity we have in mind the theory which appears in the target space of non-critical bosonic strings, or more precisely, the description of the large $N$ limit of matrix models in terms of the geometry of the eigenvalue distribution. The basic idea in this theory of gravity is to study fluctuations of a Fermi surface in a 2-dimensional phase space. The dynamical object is the area element $\omega$, representing the phase space density, which is defined to be non-zero in some region $R$ and vanishing outside. By a choice of coordinates, we can always write this area element as $\omega=d x \wedge d p$ inside $R$ and zero elsewhere. Hence the data of the theory is specified by the boundary $\partial R$, which we can consider locally as the graph of a function $p(x)$. The study of the fluctuations of the boundary is equivalent to that of fluctuations of $\omega$. Actually, in this gravity theory, one does not allow arbitrary fluctuations; rather, one considers only those which preserve the integral

$$
\oint p(x) d x=A
$$

Such fluctuations can be written $p(x)=p_{0}(x)+\partial \phi(x)$. In other words, the cohomology class of $\omega$, or "zero mode," is fixed by $A$, and the "massive modes" captured by the field $\phi(x)$ are the dynamical degrees of freedom. 
This gravity theory is related to the large $N$ limit of matrix models, where $x$ denotes the eigenvalue of the matrix and $p(x) d x$ denotes the eigenvalue density distribution. $A$ gets interpreted as the rank $N$ of the matrix. One can solve this theory using matrix models, or equivalently using $W_{\infty}$ symmetries [16].

This theory can also be viewed $[16,17]$ as the effective theory of the B model topological string on the Calabi-Yau 3-fold $u v=F(x, p)$, where $F(x, p)=0$ denotes the Fermi surface. In this language, $\omega=d x \wedge d p$ is the reduction of the holomorphic 3 -form to the $(x, p)$ plane. In the $\mathrm{B}$ model, one always fixes the cohomology class of the 3 -form; here this reduces to fixing the area $A$ as we described above.

\subsection{D gravity theory as Chern-Simons gauge theory}

Now we turn to the case of three dimensions. Pure gravity in three dimensions is topological, in a sense that it does not have propagating gravitons. In fact, we can write the usual Einstein-Hilbert action with cosmological constant $\Lambda$,

$$
S_{\text {grav }}=\int_{M} \sqrt{g}(R-2 \Lambda)
$$

in the first-order formalism,

$$
S=\int_{M} \operatorname{Tr}\left(e \wedge F+\frac{\Lambda}{3} e \wedge e \wedge e\right),
$$

where $F=d A+A \wedge A$ is the field strength of an $S U(2)$ gauge connection $A^{i}$, and $e^{i}$ is an $S U(2)$-valued 1-form on $M$. Notice that the gravity action (3.3) has the form of a BF theory, and does not involve a metric on the 3-manifold $M$. A metric (of Euclidean signature) can, however, be reconstructed from the fundamental fields - namely, given the $S U(2)$-valued 1-form $e$, one can write

$$
g_{a b}=-\frac{1}{2} \operatorname{Tr}\left(e_{a} e_{b}\right) .
$$

The equations of motion that follow from equation (3.3) are:

$$
\begin{aligned}
& D_{A} e=0, \\
& F+\Lambda e \wedge e=0 .
\end{aligned}
$$

The first equation says that $A$ can be interpreted as the spin connection for the vielbein $e^{i}$, while the second equation is the Einstein equation with cosmological constant $\Lambda$. 
Gravity in three dimensions has a well-known reformulation in terms of Chern-Simons gauge theory [18, 19],

$$
S=\int_{M} \operatorname{Tr}\left(\mathcal{A} \wedge d \mathcal{A}+\frac{2}{3} \mathcal{A} \wedge \mathcal{A} \wedge \mathcal{A}\right)
$$

where $\mathcal{A}$ is a gauge connection with values in the Lie algebra of the gauge group $G$. The gauge group $G$ is determined by the cosmological constant, and can be viewed as the isometry group of the underlying geometric structure. Specifically, in the Euclidean theory, it is either $S L(2, \mathbb{C})$, or $I S O(3)$, or $S U(2) \times S U(2)$, depending on the cosmological constant:

The equations of motion that follow from the Chern-Simons action (3.6) imply that the gauge connection $\mathcal{A}$ is flat,

$$
d \mathcal{A}+\mathcal{A} \wedge \mathcal{A}=0
$$

Writing this equation in components, one can reproduce the equations of motion (3.5). For example, if $\Lambda<0$ one can write the complex gauge field $\mathcal{A}$ as $\mathcal{A}^{k}=w^{k}+i e^{k}$. Substituting this into equation (3.7) and combining the real and imaginary terms, we recognize the equations (3.5) with $\Lambda=-1$. Finally, we note that, in the Chern-Simons theory, the gauge transformation with a parameter $\epsilon$ has the form

$$
\delta_{\epsilon} \mathcal{A}=d \epsilon-[\mathcal{A}, \epsilon]
$$

One can also describe the quantum version of $3 D$ gravity directly via various discrete models. For example, given a triangulation $\Delta$ of $M$ one can associate to each tetrahedron a quantum $6 j$-symbol and, following Turaev and Viro [20], take the state sum

$$
T V(\Delta)=\left(-\frac{\left(q^{1 / 2}-q^{-1 / 2}\right)^{2}}{2 k}\right)^{V} \sum_{j_{e}} \prod_{\text {edges }}\left[2 j_{e}+1\right]_{q} \prod_{\text {tetrahedra }}(6 j)_{q}
$$

where $V$ is the total number of vertices in the triangulation, and $[2 j+1]_{q}$ is the quantum dimension of the spin $j$ representation of $S U(2)_{q}$

$$
[n]_{q}=\frac{q^{n / 2}-q^{-n / 2}}{q^{1 / 2}-q^{-1 / 2}}
$$

One can prove [20] that the Turaev-Viro invariant is independent on the triangulation and, therefore, gives a topological invariant, $T V(M)=T V(\Delta)$. Furthermore, it has been shown by Turaev [21] and Walker [22] that the Turaev-Viro invariant is equal to the square of the partition function in $S U(2)$ Chern-Simons theory (also known as the Reshetikhin-Turaev-Witten 
invariant $[23,24])$ :

$$
T V(M)=\left|Z_{S U(2)}(M)\right|^{2}
$$

There is a similar relation between the $S L(2, \mathbb{C})$ Chern-Simons partition function and quantum invariants of hyperbolic 3-manifolds [25].

\subsection{D 2-form gravity}

In dimension four, there are several versions of "topological gravity". Here we review a theory known as 2-form gravity [26-33], which also describes the self-dual sector of loop quantum gravity [34].

We begin by writing the action for Einstein's theory in a slightly unconventional way $[26,35,36]$ :

$$
S_{4 D}=\int_{M^{4}} \Sigma^{k} \wedge F_{k}-\frac{\Lambda}{24} \Sigma^{k} \wedge \Sigma_{k}+\Psi_{i j} \Sigma^{i} \wedge \Sigma^{j}
$$

Here $A^{k}$ is an $S U(2)$ gauge field, with curvature $F^{k}=d A^{k}+\epsilon^{i j k} A^{j} \wedge A^{k}$, and $\Sigma^{k}$ is an $S U(2)$ triplet of 2 -form fields, $k=1,2,3$. The parameter $\Lambda$ will be interpreted below as a cosmological constant. Finally, $\Psi_{i j}=\Psi_{(i j)}$ is a scalar field on $M$, transforming as a symmetric tensor of $S U(2)$.

To see the connection to ordinary general relativity, one constructs a metric out of the 2 -form field $\Sigma^{k}$ as follows. The equation of motion from varying $\Psi_{i j}$ implies that $\Sigma^{k}$ obeys the constraint

$$
\Sigma^{(i} \wedge \Sigma^{j)}-\frac{1}{3} \delta^{i j} \Sigma_{k} \wedge \Sigma^{k}=0 .
$$

When equation (3.13) is satisfied the 2 -form $\Sigma^{k}$ may be reexpressed in terms of a vierbein [36],

$$
\Sigma^{k}=-\eta_{a b}^{k} e^{a} \wedge e^{b}
$$

Here $e^{a}$ are vierbein 1 -form on $M^{4}, a=1, \ldots, 4$, and $\eta_{a b}^{k}$ is the 't Hooft symbol,

$$
\eta_{a b}^{k}=\epsilon_{a b 0}^{k}+\frac{1}{2} \epsilon^{i j k} \epsilon_{i j a b}
$$

In other words,

$$
\begin{aligned}
& \Sigma^{1}=e^{12}-e^{34}, \\
& \Sigma^{2}=e^{13}-e^{42}, \\
& \Sigma^{3}=e^{14}-e^{23},
\end{aligned}
$$


where $e^{i j}=e^{i} \wedge e^{j}$. The vierbein in turn determines a metric in the usual way:

$$
g=\sum_{a=1}^{4} e^{a} \otimes e^{a} .
$$

With respect to this metric $g$, the 2 -forms $\Sigma^{k}(k=1,2,3)$ are all self-dual in the sense that $\Sigma^{k}=* \Sigma^{k}$. (This just follows from their explicit expression (3.14) in terms of the vierbein.)

One can also write the metric directly in terms of $\Sigma$ without first constructing the vierbein $[36,37]$ :

$$
\sqrt{g} g_{a b}=-\frac{1}{12} \Sigma_{a a_{1}}^{i} \Sigma_{b a_{2}}^{j} \Sigma_{a_{3} a_{4}}^{k} \epsilon^{i j k} \epsilon^{a_{1} a_{2} a_{3} a_{4}} .
$$

Having constructed the metric $g$ out of $\Sigma$, we now want to check that it obeys Einstein's equation on-shell. The equations of motion which follow from equation (3.12) are

$$
\begin{aligned}
& D_{A} \Sigma=0, \\
& F_{i}=\Psi_{i j} \Sigma^{j}+\frac{\Lambda}{12} \Sigma_{i} .
\end{aligned}
$$

The first equation $D_{A} \Sigma=0$ says that $A$ is the self-dual part of the spin connection defined by the metric $g$. The second equation then contains information about the Riemann curvature ${ }^{2}$ acting on self-dual 2-forms $\Lambda_{+}^{2}$. Namely, since the $\Sigma^{k}$ appearing on the right side are also self-dual 2-forms, the Riemann curvature maps $\Lambda_{+}^{2} \rightarrow \Lambda_{+}^{2}$. By the standard decomposition of the Riemann tensor, this implies that the trace-free part of the Ricci curvature vanishes. Then $\Psi_{i j}$ is identified with the self-dual part of the Weyl curvature, and the last term gives the trace part of the Ricci tensor, consistent with the cosmological constant $\Lambda$.

So far we have seen that the action (3.12) reproduces Einstein's theory of gravity, in the sense that the classical solutions correspond exactly to Einstein metrics on $M$ with cosmological constant $\Lambda$. Now let us consider the effect of dropping the field $\Psi_{i j}$, giving

$$
S_{4 D}=\int_{M^{4}} \Sigma^{k} \wedge F_{k}-\frac{\Lambda}{24} \Sigma^{k} \wedge \Sigma_{k}
$$

One can consider equation (3.19) as obtained by starting from equation (3.12), multiplying the last term by $\epsilon$, and then taking the $\epsilon \rightarrow 0$ limit. Just when we reach $\epsilon=0$ we seem to lose the constraint (3.13), which was the equation of motion for $\Psi_{i j}$ and was crucial for the description of $\Sigma^{k}$ in

\footnotetext{
${ }^{2}$ Here we are considering $R$ with all indices down, $R_{a b c d}$, as a symmetric map $\Lambda^{2} \rightarrow \Lambda^{2}$.
} 
terms of the vierbein. However, at $\epsilon=0$ something else happens: the action develops a large new symmetry,

$$
\begin{aligned}
\delta A_{k} & =\frac{\Lambda}{12} \theta_{k}, \\
\delta \Sigma_{k} & =D_{A} \theta_{k} .
\end{aligned}
$$

This new symmetry can be used to reimpose the constraint (3.13), so in this sense the $\epsilon \rightarrow 0$ limit is smooth and sensible to consider. The only change to the equations of motion is that the term $\Psi_{i j} \Sigma^{j}$ disappears from the right side of equation (3.18), leaving

$$
\begin{aligned}
& D_{A} \Sigma=0, \\
& F_{i}=\frac{\Lambda}{12} \Sigma_{i} .
\end{aligned}
$$

As we mentioned above, the $\Psi_{i j}$ term represents the self-dual part of the Weyl curvature; so equation (3.21) imply that the metric constructed from $\Sigma$ is not only Einstein but also has vanishing self-dual Weyl curvature. In this sense, action (3.19) gives rise to anti-self-dual Einstein manifolds,

$$
R_{a b}=\Lambda g_{a b}, \quad W_{a b c d}^{(+)}=0 .
$$

Note that such manifolds are rather rare compared to ordinary Einstein manifolds; for example, with $\Lambda>0$ there are just two smooth examples, namely $\mathbf{C P}^{2}$ and $\mathbf{S}^{4}$ [38]. With $\Lambda=0$ the solutions are hyperkähler metrics in 4 dimensions; these are target spaces for the $\mathcal{N}=2$ string (or equivalently the $\mathcal{N}=4$ topological string), which provides a completion of the self-dual gravity theory in that case.

\subsection{D form theories: Kähler and Kodaira-Spencer gravity}

In dimension 6, two different form theories of gravity arise in $(\mathcal{N}=2)$ topological string theory. One, known as the Kähler gravity theory [39], describes the target space gravity (string field theory) of the topological A model. The second theory, called the Kodaira-Spencer theory of gravity [7], is the string field theory of the topological B model and describes variations of the complex structure. Below we review each of these theories in turn.

We begin with the $\mathrm{B}$ model. The basic field of the Kodaira-Spencer gravity theory is a vector-valued 1-form field $A$, for which the action is given by $[7]$

$$
S_{\mathrm{KS}}=\frac{1}{2} \int_{M} A^{\prime} \frac{1}{\partial} \bar{\partial} A^{\prime}+\frac{1}{6} \int_{M}(A \wedge A)^{\prime} \wedge A^{\prime}
$$

Here, we use the standard notation $A^{\prime}:=\left(A \cdot \Omega_{0}\right)$ for the product with the background holomorphic $(3,0)$ form. The field $A$ then defines a variation of 
$\Omega$, given by the formula

$$
\Omega=\Omega_{0}+A^{\prime}+(A \wedge A)^{\prime}+(A \wedge A \wedge A)^{\prime} .
$$

This expression for the variation of $\Omega$ follows from its local expression in complex coordinates, $\Omega=\Omega_{i j k} d z^{i} \wedge d z^{j} \wedge d z^{k}$, where $A$ is interpreted as giving a variation of the 1 -form $d z^{i}$ :

$$
d z^{i} \mapsto d z^{i}+A_{\bar{j}}^{i} d \bar{z}^{\bar{j}}
$$

In order that the "non-local term" $\frac{1}{\partial} \bar{\partial} A^{\prime}$ in the action (3.23) make sense, $A$ is not allowed to be an arbitrary vector-valued 1-form; rather, there is a constraint

$$
\partial A^{\prime}=0
$$

Using this constraint we write

$$
A^{\prime}=x+\partial \phi, \quad x \in H^{2,1}(M, \mathbb{C}) .
$$

Here the harmonic $x \in H^{2,1}(M, \mathbb{C})$ represents the massless modes (moduli) of $\Omega$, which are frozen in the Kodaira-Spencer theory, while $\phi \in \Omega^{1,1}(M, \mathbb{C})$ represents the massive modes, which are the dynamical degrees of freedom. Substituting equation (3.27) into equation (3.23), we can write the KodairaSpencer action without non-local terms:

$$
S_{\mathrm{KS}}=\frac{1}{2} \int_{M} \partial \phi \wedge \bar{\partial} \phi+\frac{1}{6} \int_{M}(A \wedge A)^{\prime} \wedge A^{\prime}
$$

The equation of motion that follows from the action (3.28) is

$$
\bar{\partial} A^{\prime}+\partial(A \wedge A)^{\prime}=0
$$

Using equations (3.26) and (3.29) together one finds that the holomorphic 3 -form (3.24) is closed on-shell,

$$
d \Omega=0 .
$$

Hence solutions to the Kodaira-Spencer field equations correspond to deformations $\Omega$ of the holomorphic 3 -form $\Omega_{0}$.

When we view $\phi$ as the dynamical degree of freedom, we must note that it has a large shift symmetry,

$$
\phi \mapsto \phi+\epsilon,
$$

where $\partial \epsilon=0$. This symmetry can be used to set the anti-holomorphic part of $\phi$ to zero, i.e., $\bar{\partial} \phi=0$. In other words, $\phi$ should be viewed as the analog of the chiral boson in 2 dimensions; in this sense, the Kodaira-Spencer theory is really a chiral theory. In fact, in the local geometry we discussed in Section $3.1, \phi$ gets identified with a chiral boson on the Riemann surface $F(x, p)=0$.

Although $A^{\prime}$ and the Kodaira-Spencer action depend on $\partial \phi$ rather than on $\phi$ itself, it turns out that D1-branes of the B model are charged under 
$\phi$ directly. To see this, consider a D1-brane wrapped on a 2-cycle $E$ which moves to another 2-cycle $E^{\prime}$. There is a 3 -chain $C$ which interpolates between $E$ and $E^{\prime}$, and the variation of the action is given by (absorbing the string coupling constant into $\Omega$ )

$$
\delta S=\int_{C} \Omega=\int_{C} \partial \phi=\int_{C} d \phi=\int_{E} \phi-\int_{E^{\prime}} \phi .
$$

This coupling also explains the fact that a D1-brane is a source for $\Omega$ (and hence shifts the integral of $\Omega$ on a 3 -cycle linking it). Namely, including such a source localized along $E$ would modify the equations of motion to [16]

$$
\bar{\partial} A^{\prime}=\bar{\partial} \partial \phi=\delta_{E}^{4},
$$

so that the kinetic term $\phi \partial \bar{\partial} \phi$ from equation (3.28) becomes precisely $\int_{E} \phi$.

The fact that D1-branes couple to $\phi$ has an important consequence: there is an extra $H^{1,1}(M, \mathbb{C})$ worth of degrees of freedom in $\phi$, corresponding to the freedom to shift $\phi$ by a harmonic form $b$, which does not affect $A^{\prime}$ but does figure into non-perturbative aspects of the B model. Namely, the amplitudes involving D1-brane instantons, which should presumably be included in the non-perturbative definition of the B model, are sensitive to this shift. Thus the partition function of the $\mathrm{B}$ model is non-perturbatively a function both of $x \in H^{2,1}(M)$ and of $b \in H^{1,1}(M, \mathbb{C})$. The necessity of the extra field $b$ was also recently noted in [40].

As we will discuss later in more detail, it is natural that in a nonperturbative definition the periods of $\Omega$ are quantized in units of $g_{s}$. There is an overall $1 / g_{s}^{2}$ in front of the closed string action, so this will then give the appropriate $1 / g_{s}$ coupling of the field $\phi$ to the D-branes. Because of this flux quantization, the corresponding "Wilson lines" $b$ will be naturally periodic or $\mathbb{C}^{*}$ variables.

Having discussed the Kodaira-Spencer theory, let us now turn to another 6-dimensional form theory of gravity, namely the Kähler gravity theory, which describes variations of the Kähler structure on $M$. Its action is [39, 41]

$$
S_{\text {Kahler }}=\int_{M}\left(\frac{1}{2} K \frac{1}{d^{c \dagger}} d K+\frac{1}{3} K \wedge K \wedge K\right),
$$

where $K$ is a variation of the (complexified) Kähler form on $M$, and $d^{c}=$ $\partial-\bar{\partial}$. The Kähler gravity action (3.34) is invariant under gauge transformations of the form

$$
\delta_{\alpha} K=d \alpha-d^{c \dagger}(K \wedge \alpha)
$$

where $\alpha$ is a 1 -form on $M$, such that $d^{c \dagger} \alpha=0$. The equations of motion in the Kähler gravity theory are

$$
d K+d^{c \dagger}(K \wedge K)=0 .
$$


As in the Kodaira-Spencer theory, we can decompose $K$ into massless and massive modes,

$$
K=x+d^{c \dagger} \gamma, \quad x \in H^{1,1}(M, \mathbb{C})
$$

where $x \in H^{1,1}(M, \mathbb{C})$ represents the Kähler moduli, which are not integrated over, and $\gamma \in \Omega^{3}(M)$ contains the massive modes of $K$. Using equation (3.37), we can write the Kähler gravity action (3.34) without non-local terms,

$$
S_{\text {Kahler }}=\int_{M}\left(\frac{1}{2} d \gamma \wedge d^{c \dagger} \gamma+\frac{1}{3} K \wedge K \wedge K\right) \text {. }
$$

Just as in the B model, Lagrangian D-branes of the A model are charged under $\gamma$, implying that these branes are sources for $K$ and hence modify the integral of $K$ on 2-cycles which link them. This also implies that the partition function of the A model depends non-perturbatively on the choice of a cohomology class in $H^{3}(M)$ as well as on $x \in H^{2}(M)$. Here the same remarks about flux quantization hold as in the $\mathrm{B}$ model.

\section{Hitchin's action functionals}

In the previous section, we discussed various form theories of gravity which have appeared previously in the physics literature. Now we turn to some new ones. We will describe actions constructed by Hitchin $[10,42]$ for which the equations of motion yield special geometric structures: either holomorphic 3forms $\Omega$ and symplectic structures $k$ in 6 dimensions or $G_{2}$ holonomy metrics in 7 dimensions. As for the form theories we considered above, the classical fields in these theories are real $p$-forms, from which the desired geometric structures can be reconstructed. In 6 dimensions one has a 3 -form $\rho$ and a 4 -form $\sigma$; these forms will be interpreted as $\rho=\operatorname{Re} \Omega, \sigma=\frac{1}{2} k \wedge k$. In 7 dimensions, one just has a single 3-form $\Phi$ (or its dual 4-form $G$ ), interpreted as the associative 3 -form (respectively coassociative 4 -form) of the $G_{2}$ metric (figure 1).

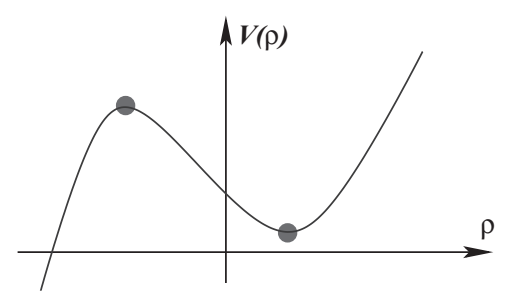

Figure 1: Critical points of volume functionals define special geometric structures on $X$. 
These action functionals have been used in the physics literature to construct new metrics with special holonomy [43-45]. In the present context, they should be regarded as effective actions for gravity theories. In 6 dimensions, we will see in Section 5 that these gravity theories are related to topological strings. The 7-dimensional action defines a new gravity theory which we identify as the low energy limit of topological M-theory.

\subsection{Special holonomy manifolds and calibrations}

In this subsection, we briefly review the notion of special holonomy, which plays an important role in supersymmetric string compactifications, and which we expect to be important for topological string/membrane theories. In particular, we emphasize that the geometric structure on a special holonomy manifold $X$ can be conveniently characterized by certain $p$-forms, invariant under the holonomy group, $\operatorname{Hol}(X)$.

Recall that for any $n$-dimensional Riemannian manifold $X$ we have

$$
\operatorname{Hol}(X) \subseteq S O(n)
$$

The manifolds with special (reduced) holonomy are characterized by the condition $\operatorname{Hol}(X) \subset S O(n)$. Below we list some examples of special holonomy manifolds that will be relevant in what follows.

All of these structures can be characterized by the existence of a covariantly constant spinor,

$$
\nabla \xi=0
$$

The existence of this $\xi$ guarantees that superstring compactification on $X$ preserves some fraction (also listed in the above table) of the original 32 supercharges, which is what makes such manifolds useful in string theory.

Another characteristic property of special holonomy manifolds is the existence of invariant forms, known as calibrations. Using the covariantly constant spinor $\xi$ one can construct a $p$-form on $X$,

$$
\omega^{(p)}=\xi^{\dagger} \gamma_{i_{1} \ldots i_{p}} \xi
$$

By construction, such forms are covariantly constant and invariant under $\operatorname{Hol}(X)$. They are non-trivial only for special values of $p$ : see table 1 for a list of the invariant forms on manifolds of $S U(3), G_{2}$, and $\operatorname{Spin}(7)$ holonomy. These invariant forms play an important role in geometry and physics; in particular, they can be used to characterize minimal (supersymmetric) cycles in special holonomy manifolds. Indeed, if $S \subset X$ is a minimal submanifold 
Table 1: Examples of special holonomy manifolds

\begin{tabular}{lcccll}
\hline Metric & Holonomy & $n$ & SUSY & Invariant & $p$-forms \\
\hline Calabi-Yau & $S U(3)$ & 6 & $1 / 4$ & $\begin{array}{l}p=2: K \\
p=3: \Omega\end{array}$ & (Kähler) \\
Exceptional & $G_{2}$ & 7 & $1 / 8$ & $\begin{array}{l}p=3: \Phi \\
p=4: * \Phi\end{array}$ & (associative) \\
& (coassociative) & & & \\
& $\operatorname{Spin}(7)$ & 8 & $1 / 16$ & $p=4: \Psi$ & (Cayley) \\
\hline
\end{tabular}

of real dimension $p$, then its volume can be determined by integrating the invariant form $\omega^{(p)}$ over $S$,

$$
\operatorname{Vol}(S)=\int_{S} \omega^{(p)}
$$

Such a manifold $S$ is called calibrated, and the form $\omega^{(p)}$ is called a calibration. Notice the simplification that has occurred here. Ordinarily, in order to compute the volume, $\operatorname{Vol}(S)=\int d^{p} x \sqrt{g}$, we need to know the metric $g$; but the volume of a calibrated submanifold $S$ is given by the simple formula (4.4) which does not involve the explicit form of the metric.

This phenomenon is a prototype of various situations in which the important geometric data can be characterized by differential forms rather than by a metric. This is essentially the same principle that was underlying the constructions of Section 3, where we discussed form theories of gravity in which the space-time geometry is encoded in tensor forms and/or gauge fields.

To illustrate further the idea that forms can capture the geometry, let us consider an example which will play an important role below. Let $X$ be a manifold with $G_{2}$ holonomy. The existence of a $G_{2}$ holonomy metric is equivalent to the existence of an associative 3 -form, $\Phi$, which is closed and co-closed,

$$
\begin{aligned}
& d \Phi=0 \\
& d * \Phi=0
\end{aligned}
$$

and which can be written in terms of an orthonormal vielbein $e^{i}, i=1, \ldots, 7$, as

$$
\Phi=\frac{1}{3 !} \psi_{i j k} e^{i} \wedge e^{j} \wedge e^{k} .
$$

Here $\psi_{i j k}$ are the structure constants of the imaginary octonions: $\sigma_{i} \sigma_{j}=$ $-\delta_{i j}+\psi_{i j k} \sigma_{k}, \sigma_{i} \in \operatorname{Im}(\mathbb{O})$. Conversely, writing $\Phi$ locally in the form (4.6) 
defines a metric $g$ by the formula

$$
g=\sum_{i=1}^{7} e^{i} \otimes e^{i} .
$$

This $g$ can be written more explicitly by first defining

$$
B_{j k}=-\frac{1}{144} \Phi_{j i_{1} i_{2}} \Phi_{k j_{3} j_{4}} \Phi_{j_{5} j_{6} j_{7}} \epsilon^{j_{1} \ldots j_{7}},
$$

in terms of which the metric has a simple form,

$$
g_{i j}=\operatorname{det}(B)^{-1 / 9} B_{i j} .
$$

Evaluating the determinant of $B_{i j}$, we get $\operatorname{det}(g)=\operatorname{det}(B)^{2 / 9}$, so equation (4.9) can be written in a more convenient form,

$$
\sqrt{g} g_{j k}=-\frac{1}{144} \Phi_{j i_{1} i_{2}} \Phi_{k i_{3} i_{4}} \Phi_{i_{5} i_{6} i_{7}} \epsilon^{i_{1} \ldots i_{7}} .
$$

Notice that even if the 3 -form $\Phi$ does not obey equation (4.5), we can still use equation (4.10) to construct a metric on $X$ from $\Phi$, as long as the 3 -form $\Phi$ is non-degenerate in a suitable sense. (Of course, this metric will not have $G_{2}$ holonomy unless equation (4.5) is satisfied.) This construction naturally leads us to the notion of stable forms, which we now discuss.

\subsection{Stable forms}

Following [10], in this section, we review the construction of action principles from $p$-forms. It is natural to define the action of a $p$-form $\rho$ on a manifold $X$ as a volume form $\phi(\rho)$ integrated over $X$. One might think that such a $\phi(\rho)$ is hard to construct, as one might have in mind the usual wedge product of $\rho$ with itself, which gives a non-zero top-form only in rather special cases. In fact, this is not the only way to build a volume form out of $p$-forms; as we will see, all the actions of interest for us turn out to involve a volume element constructed in a rather non-trivial way from the $p$-form. For example, on a 7-manifold with $G_{2}$ holonomy, the volume form cannot be constructed as a wedge product of the associative 3 -form $\Phi$ with itself; nevertheless, one can define $\phi(\Phi)$ as a volume form for the metric (4.9) constructed from $\Phi$, as we will discuss in detail later.

The most general way to construct a volume form $\phi(\rho)$ from a $p$-form $\rho$ is as follows: contract a number of $\rho_{i_{1}, \ldots, i_{p}}$ 's with a number of epsilon tensors $\epsilon^{i_{1}, \ldots, i_{n}}$, to obtain some $W(\rho)$. Suppose we use $k$ epsilon tensors in $W$; then $W$ transforms as the $k$ th power of a volume, and we can define 
$\phi(\rho)=W(\rho)^{1 / k}$ which is a volume form. It is easy to see that $\phi(\rho)$ scales as an $n / p$ th power of $\rho$ (if $W$ has $q$ factors of $\rho$ it will need $k=p q / n$ factors of $\epsilon$ ).

Given such a volume form, one can define the action to be the total volume,

$$
V(\rho)=\int_{X} \phi(\rho)
$$

This $V(\rho)$ is a homogeneous function of $\rho$ of degree $\frac{n}{p}$ :

$$
V(\lambda \rho)=\lambda^{\frac{n}{p}} V(\rho) \quad \lambda \in \mathbb{R} .
$$

In this paper, we will not be interested in arbitrary ways of putting together $\rho$ and $\epsilon$ symbols to make $\phi(\rho)$. Rather, we will focus on cases in which there exists a notion of "generic" $p$-form. In such cases, the generic $p$-form $\rho$ defines an interesting geometric structure (e.g., an almost complex structure or a $G_{2}$ structure) even without imposing any additional constraints. Hence arbitrary variations of $\rho$ can be thought of as variations of this structure, and as we will see, critical points of $V(\rho)$ imply integrability conditions on these geometric structures.

The notion of genericity we have in mind is known as stability, as described in [42] and reviewed below. The requirement of stability has drastically different consequences depending on the dimension $n$ of the manifold and the degree $p$ of the form. In most cases, as we will see, there are no stable forms at all; but for certain special values of $n$ and $p$, stable forms can exist. Moreover, all the calibrations in 6 and 7 dimensions that we discussed earlier turn out to be stable forms. This deep "coincidence" makes the technology of stable forms a useful tool for the study of special holonomy. Nevertheless, possessing a stable form is far less restrictive than the requirement of special holonomy, needed for supersymmetry.

Let us now define the notion of stability precisely. Write $V$ for the tangent space at a point $x$, so the space of $p$-forms at $x$ is $\Lambda^{p} V^{*}$. Then a $p$-form $\rho$ is said to be stable at $x$ if $\rho(x)$ lies in an open orbit of the $G L(V)$ action on $\Lambda^{p} V^{*}$. We call $\rho$ a stable form if $\rho$ is stable at every point. In other words, $\rho$ is stable if all forms in a neighborhood of $\rho$ are equivalent to $\rho$ by a local $G L(n)$ action.

Some special cases of stability are easy to understand. For example, there are no stable 0 -forms, because under coordinate transformations the value of a function does not change. On the other hand, any non-zero $n$-form is stable, because by a linear transformation one can always map any volume form to any other one. Similarly, any non-zero 1-form or $(n-1)$-form is stable. 
A less trivial case of stability is that of a 2-form, on a manifold of even dimension. In this case, viewing the 2 -form as an antisymmetric matrix, stability just means that the determinant is non-zero; namely, this is the usual characterization of a presymplectic form, and any such form can be mapped to any other by a linear transformation, so they are indeed stable. Given such a stable form, we can now construct its associated volume form: namely, we write $\phi(\omega)=\omega^{n / 2}$. Note that the stability of $\omega$ is equivalent to $\phi(\omega) \neq 0$.

To understand the geometric structures defined by stable forms, it is useful to study the subgroup of $G L(n)$ which fixes them. In the case of a stable 2-form in even dimension this stabilizer is $S p(n)$, corresponding to the fact that the 2-form defines a presymplectic structure. More generally, given a stable $p$-form, we can easily compute the dimension of the stabilizer: it is simply the dimension of $G L(n)$ minus the dimension of the space of $p$-forms. In the case $p=2$, this counting gives $n^{2}-\frac{n(n-1)}{2}=n(n+1) / 2$, as expected.

Next we consider the case $p=3$. The dimension of the space of 3 -forms is

$$
\operatorname{dim} \Lambda^{3} V^{*}=n(n-1)(n-2) / 6 .
$$

Already at this stage we see that there cannot be any stable 3 -forms for large $n$, because $\operatorname{dim} G L(n)=n^{2}$ has a slower growth than $n^{3} / 6$, so that $G L(n)$ cannot act locally transitively on the space of 3-forms. However, for $p=3$ and small enough $n$ the stability condition can be met. We have already discussed the cases $n=3,4$, and stable 3 -forms also exist for $n=6,7,8$. These special cases lead to interesting geometric structures; for example, consider the case $p=3, n=7$. Here the dimension counting gives

$$
\begin{aligned}
\operatorname{dim} G L(V)=n^{2}= & 49, \\
\operatorname{dim} \Lambda^{p} V^{*}=\frac{n !}{p !(n-p) !}= & 35, \\
& 14=\operatorname{dim} G_{2} .
\end{aligned}
$$

Indeed, the stabilizer of the 3 -form in this case is $G_{2}$, so a stable 3 -form in seven dimensions defines a $G_{2}$ structure.

As we just discussed for $p=3$, generically $\operatorname{dim} \Lambda^{p} V^{*}$ is much larger than $\operatorname{dim} G L(V)=n^{2}$. Hence stable forms exist only for special values of $n$ and $p$ [42]. The cases of interest for us in this paper are $n=7$ with $p=3,4$ and $n=6$ with $p=3,4$. We now turn to the construction of volume functionals from stable forms in these cases. 


\subsection{3-form and 4-form actions in 6D}

We begin with the 6-dimensional case. In this case Hitchin constructed two action functionals $V_{H}(\rho)$ and $V_{S}(\sigma)$, depending respectively on a 3 -form $\rho$ and 4-form $\sigma$. When extremized, $V_{H}$ and $V_{S}$ yield, respectively, holomorphic and symplectic structures on $M$. In this section we introduce these action functionals and describe some of their properties.

Let us first construct $V_{S}(\sigma)$. Suppose $\sigma$ is a stable 4-form; the stability condition in this case means there exists $k$ such that $\sigma= \pm \frac{1}{2} k \wedge k$. We consider the + case here. Interpreting this $k$ as a candidate symplectic form, $V_{S}(\sigma)$ is defined to be the symplectic volume of $M$ :

$$
V_{S}(\sigma)=\frac{1}{6} \int_{M} k \wedge k \wedge k
$$

This action can also be written directly in terms of $\sigma$ :

$$
\begin{aligned}
V_{S}(\sigma) & =\frac{1}{6} \int_{M} \sigma^{3 / 2} \\
& =\int_{M} \sqrt{\frac{1}{384} \sigma_{a_{1} a_{2} b_{1} b_{2}} \sigma_{a_{3} a_{4} b_{3} b_{4}} \sigma_{a_{5} a_{6} b_{5} b_{6}} \epsilon^{a_{1} a_{2} a_{3} a_{4} a_{5} a_{6}} \epsilon^{b_{1} b_{2} b_{3} b_{4} b_{5} b_{6}}},
\end{aligned}
$$

where $\epsilon^{a_{1} \ldots a_{6}}$ is the Levi-Civita tensor in six dimensions. As discussed before, the need to take a square root arises because to define a volume form we need to have exactly one net $\epsilon$ tensor.

We will be considering $V_{S}(\sigma)$ as the effective action of a gravity theory in 6 dimensions. We treat $\sigma$ as a 4 -form field strength for a 3 -form gauge field $\gamma$ : in other words, we hold the cohomology class of $\sigma$ fixed,

$$
\begin{aligned}
& {[\sigma] \in H^{4}(M, \mathbb{R}) \quad \text { fixed, i.e., }} \\
& \sigma=\sigma_{0}+d \gamma
\end{aligned}
$$

where $d \sigma_{0}=0$. Now we want to find the classical solutions, i.e., critical points of $V_{S}(\sigma)$. Write

$$
V_{S}(\sigma)=\frac{1}{3} \int_{M} \sigma \wedge k=\frac{1}{3} \int_{M}\left(\sigma_{0}+d \gamma\right) \wedge k .
$$

Varying $\gamma$ then gives a term

$$
\delta V_{S}=\frac{1}{3} \int_{M} d(\delta \gamma) \wedge k=-\frac{1}{3} \int_{M} \delta \gamma \wedge d k .
$$

This is not the whole variation of $V_{S}$, because $k$ also depends on $\sigma$; but it turns out that the extra term from the variation of $k$ is just $1 / 2$ of equation (4.19). This is a consequence of the fact (4.12) that $V_{S}(\sigma)$ is 
homogeneous as a function of $\sigma$. Altogether, the condition that $V_{S}(\sigma)$ is extremal under variations of $\gamma$ is simply

$$
d k=0 .
$$

Hence the classical solutions of the gravity theory based on $V_{S}(\sigma)$ give symplectic structures on $M$.

Having discussed $V_{S}(\sigma)$, we now turn to $V_{H}(\rho)$. Suppose $\rho$ is a stable 3 -form. Provided that $\rho$ is "positive" in a sense to be defined below, it is fixed by a subgroup of $G L(6, \mathbb{R})$ isomorphic to (two copies of) $S L(3, \mathbb{C})$; this $\rho$, therefore, determines a reduction of $G L(6)$ to $S L(3, \mathbb{C})$, which is the same as an almost complex structure on $M$. More concretely, we can find three complex 1-forms $\zeta_{i}$ for which

$$
\rho=\frac{1}{2}\left(\zeta_{1} \wedge \zeta_{2} \wedge \zeta_{3}+\overline{\zeta_{1}} \wedge \overline{\zeta_{2}} \wedge \overline{\zeta_{3}}\right),
$$

and these $\zeta_{i}$ determine the almost complex structure. If locally there exist complex coordinates such that $d z_{i}=\zeta_{i}$, then the almost complex structure is integrable (it defines an actual complex structure.) Whether it is integrable or not, we can construct a $(3,0)$ form on $M$, namely

$$
\Omega=\zeta_{1} \wedge \zeta_{2} \wedge \zeta_{3}
$$

This $\Omega$ can also be written

$$
\Omega=\rho+i \widehat{\rho}(\rho)
$$

where $\widehat{\rho}$ is defined as

$$
\widehat{\rho}=-\frac{i}{2}\left(\zeta_{1} \wedge \zeta_{2} \wedge \zeta_{3}-\overline{\zeta_{1}} \wedge \overline{\zeta_{2}} \wedge \overline{\zeta_{3}}\right)
$$

Through equations (4.21), (4.22), and (4.24), we can regard $\Omega$ and $\widehat{\rho}$ as functions of $\rho$. The integrability condition is equivalent to the requirement that $d \Omega=0$.

So far we have explained how a positive stable 3-form $\rho$ determines an almost complex structure and a holomorphic 3 -form $\Omega$. Now $V_{H}(\rho)$ is defined to be the holomorphic volume:

$$
V_{H}(\rho)=-\frac{i}{4} \int_{M} \Omega \wedge \bar{\Omega}=\frac{1}{2} \int_{M} \widehat{\rho}(\rho) \wedge \rho .
$$

More concretely, using results from [10], this can be written

$$
V_{H}(\rho)=\int_{M} \sqrt{-\frac{1}{6} K_{a}^{b} K_{b}^{a}}
$$


where $^{3}$

$$
K_{a}^{b}:=\frac{1}{12} \rho_{a_{1} a_{2} a_{3}} \rho_{a_{4} a_{5} a} \epsilon^{a_{1} a_{2} a_{3} a_{4} a_{5} b} .
$$

As we did with $V_{S}$, we want to regard $V_{H}$ as the effective action of some gravity theory in which $\rho$ is treated as a field strength. So we start with a closed stable 3 -form $\rho_{0}$ and allow it to vary in a fixed cohomology class,

$$
\rho=\rho_{0}+d \beta .
$$

Then varying $\beta$, we obtain two terms, one from the variation of $\rho$ and one from the variation of $\widehat{\rho}(\rho)$. As in the case of $V_{S}$, the homogeneity of $V_{H}(\rho)$ implies that these two terms are equal, and they combine to give

$$
\delta V_{H}=\int_{M} d(\delta \gamma) \wedge \widehat{\rho}=-\int_{M} \delta \gamma \wedge d \widehat{\rho} .
$$

Hence the equation of motion is

$$
d \widehat{\rho}=0 .
$$

From equation (4.28) we also have $d \rho=0$. So altogether on-shell we have $d \Omega=0$, which is the condition for integrability of the almost complex structure, as explained above. In this sense, $V_{H}(\rho)$ is an action which generates complex structures together with holomorphic 3 -forms.

Finally, let us make one more observation about the functionals $V_{H}$ and $V_{S}$. So far we have discussed them separately, but since they both exist on a 6 -manifold $M$, it is natural to ask whether the structures they define are compatible with one another. Specifically, we would like to interpret $k$ as the Kähler form on $M$, in the complex structure determined by $\Omega$. For this interpretation to be consistent, there are two conditions which must be satisfied:

$$
k \wedge \rho=0,
$$

and

$$
2 V_{S}(\sigma)=V_{H}(\rho) .
$$

The condition (4.30) expresses the requirement that $k$ is of type $(1,1)$ in the complex structure determined by $\Omega$, while (4.31) is the equality of the volume forms determined independently by the holomorphic and symplectic structures. Requiring equations (4.30) and (4.31), $\Omega$ and $k$ together give an $S U(3)$ structure on $M$; if in addition $d \Omega=0, d k=0$, then $M$ is Calabi-Yau, with $\Omega$ as holomorphic 3-form and $k$ as Kähler form. When we discuss the Hamiltonian quantization of topological M-theory in Section 7, we will see one way in which these constraints could arise naturally.

\footnotetext{
${ }^{3}$ Having written this formula, we can now explain the positivity condition on $\rho$ to which we alluded earlier: the square root which appears in equation (4.26) should be real.
} 


\subsection{3-Form and 4-form actions in $7 \mathrm{D}$}

Now let us discuss the 7-dimensional case. We will construct two functionals $V_{7}(\Phi), V_{7}(G)$ depending on a 3-form or 4-form, respectively, both of which generate $G_{2}$ holonomy metrics on a 7 -manifold $X$.

The two cases are very similar to one another; we begin with the 3form case. A stable 3 -form $\Phi \in \Omega^{3}(X, \mathbb{R})$ determines a $G_{2}$ structure on $X$, because $G_{2}$ is the subgroup of $G L(7, \mathbb{R})$ fixing $\Phi$ at each point, as we explained in Section 4.2. There we gave the explicit expression for the metric $g$ in terms of the 3 -form $\Phi$ :

$$
g_{j k}=B_{j k} \operatorname{det}(B)^{-1 / 9},
$$

where from equation (4.8),

$$
B_{j k}=-\frac{1}{144} \Phi_{j i_{1} i_{2}} \Phi_{k i_{3} i_{4}} \Phi_{i_{5} i_{6} i_{7}} \epsilon^{i_{1} \ldots i_{7}}
$$

We can thus introduce a volume functional, $V_{7}(\Phi)$, which is simply the volume of $X$ as determined by $g$ :

$$
V_{7}(\Phi)=\int_{X} \sqrt{g_{\Phi}}=\int_{X}(\operatorname{det} B)^{1 / 9}
$$

where $B$ is the symmetric tensor defined in equation (4.33).

In order to identify the critical points of the action functional (4.34), it is convenient to rewrite it slightly. For this we use the fact that since $\Phi$ determines the metric, it also determines any quantity which could be derived from the metric; in particular, it determines a Hodge $*$-operator, which we write $*_{\Phi}$. Using this operator we can rewrite equation (4.34) as

$$
V_{7}(\Phi)=\int_{X} \Phi \wedge *_{\Phi} \Phi
$$

As we did in the 6-dimensional cases, we regard $\Phi$ as a field strength for a 2 -form gauge potential; in other words, we assume $\Phi$ is closed and vary it in a fixed cohomology class:

$$
\begin{aligned}
& {[\Phi] \in H^{3}(X, \mathbb{R}) \quad \text { fixed, i.e., }} \\
& \Phi=\Phi_{0}+d B,
\end{aligned}
$$

with $d \Phi_{0}=0$, and $B$ an arbitrary real 2-form on $X$. Using the homogeneity property (4.12) of the volume functional (4.35), we find

$$
\frac{\delta V_{7}(\Phi)}{\delta \Phi}=\frac{7}{3} *_{\Phi} \Phi
$$


Hence critical points of $V_{7}(\Phi)$ in a fixed cohomology class give 3-forms which are closed and co-closed,

$$
\begin{aligned}
& d \Phi=0, \\
& d *_{\Phi} \Phi=0 .
\end{aligned}
$$

These are precisely the conditions under which $\Phi$ is the associative 3 -form for a $G_{2}$ holonomy metric on $X$.

So far we have discussed stable 3 -forms, but the $G_{2}$ holonomy condition can also be obtained from a dual action based on a stable 4 -form $G$,

$$
V_{7}(G)=\int_{X} G \wedge *_{G} G
$$

It is this $V_{7}(G)$ which we propose to identify as the effective action of the 7-dimensional topological M-theory. As in the previous cases, we vary the 4-form $G$ in a fixed cohomology class:

$$
\begin{aligned}
& {[G] \in H^{4}(X, \mathbb{R}) \text { fixed, i.e., }} \\
& G=G_{0}+d \Gamma,
\end{aligned}
$$

where $\Gamma$ is an arbitrary real 3 -form on $X$, and $G_{0}$ is closed, $d G_{0}=0$. The condition that equation (4.39) is extremal under variations of $\Gamma$ is then simply

$$
\begin{aligned}
& d G=0, \\
& d *_{G} G=0,
\end{aligned}
$$

which is again the condition equation (4.38) that $X$ has $G_{2}$ holonomy, now written in terms of the coassociative 4 -form $G=*_{\Phi} \Phi$. Just as with $\Phi$, one can reconstruct the $G_{2}$ holonomy metric from $G$, using the expression of $G$ in terms of an orthonormal vielbein,

$$
G=e^{7346}-e^{7126}+e^{7135}-e^{7425}+e^{1342}+e^{5623}+e^{5641} .
$$

The 4-form action (4.39) can also be written in a slightly different form. One introduces a fixed basis of the space $\wedge^{2} V$ of bivectors in 7 dimensions: $e_{a}^{i j}=-e_{a}^{j i}$. Here $i, j=1, \ldots, 7$ and $a=1, \ldots, 21$, since the space of bivectors is 21-dimensional. Then define the matrix $Q_{a b}$ by

$$
Q_{a b}=\frac{1}{2} e_{a}^{i j} e_{b}^{k l} G_{i j k l} .
$$

The action for $G$ can then be written as

$$
V_{7}(G)=\int_{X}(\operatorname{det} Q)^{\frac{1}{12}}
$$

Note that since $Q$ is a matrix of rank 21, this action is indeed homogeneous of degree $\frac{21}{12}=\frac{7}{4}$ in $G$. It is a tempting thought that this action could be interpreted as a membrane version of Born-Infeld obtained by integrating 
out open (topological) membranes, since the exponent $\frac{1}{12}$ reminds one of a stringy one-loop determinant.

\subsection{Hamiltonian flow}

Now we shift gears to discuss a bridge between the $S U(3)$ structures and $G_{2}$ holonomy metrics considered in the last two subsections: we will describe a flow which constructs $G_{2}$ holonomy metrics from the $S U(3)$ structures which appeared there. This flow is essentially a Hamiltonian version of the Lagrangian field theories described in Section 4.4.

Suppose given a 6-manifold $M$, with stable forms $\rho \in \Omega^{3}(M, \mathbb{R})$ and $\sigma \in$ $\Omega^{4}(M, \mathbb{R})$. As we discussed above, if $\rho$ and $\sigma$ satisfy the compatibility conditions (4.30) and (4.31), they define an $S U(3)$ structure on $M$ and a corresponding metric. If $\rho$ and $\sigma$ are also both closed, one can extend the metric on $M$ uniquely to a $G_{2}$ holonomy metric on $X=M \times(a, b)$ for some interval $(a, b)$ (figure 2). Hitchin gave an elegant construction of this metric [10]: one takes the given $\rho$ and $\sigma$ as "initial data" on $M \times\left\{t_{0}\right\}$ and then lets $\rho$ and $\sigma$ flow according to

$$
\begin{array}{r}
\frac{\partial \rho}{\partial t}=d k \\
\frac{\partial \sigma}{\partial t}=k \wedge \frac{\partial k}{\partial t}=-d \widehat{\rho} .
\end{array}
$$

Here, as usual, $\sigma=\frac{1}{2} k \wedge k$, and $t$ is the "time" direction normal to $M$.

The evolution equations (4.45) are equivalent to the $G_{2}$ holonomy conditions (4.38) for the 3 -form

$$
\Phi=\rho(t)+k(t) \wedge d t .
$$

Moreover, equations (4.45) can be interpreted as Hamiltonian flow equations. Namely, one considers the variations of $\sigma$ and $\rho$ as spanning a phase

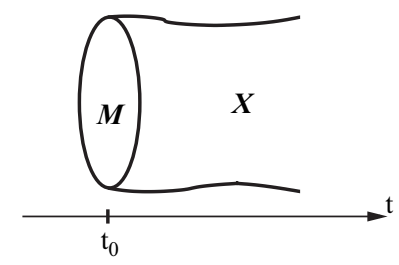

Figure 2: $\mathrm{A} G_{2}$ structure on the local geometry $X=M \times(a, b)$ can be viewed as a Hamiltonian flow. 
space $\Omega_{\text {exact }}^{4}(M, \mathbb{R}) \times \Omega_{\text {exact }}^{3}(M, \mathbb{R})$; writing $\delta \sigma=d \beta$ and $\delta \rho=d \alpha$, the symplectic pairing on the phase space is

$$
\langle\delta \sigma, \delta \rho\rangle=\int \alpha \wedge d \beta=-\int \beta \wedge d \alpha .
$$

Then equations (4.45) are precisely the Hamiltonian flow equations with respect to

$$
H=2 V_{S}(\sigma)-V_{H}(\rho),
$$

where $V_{H}(\rho)$ and $V_{S}(\sigma)$ are the volume functionals (4.15) and (4.25) which we used to obtain $S U(3)$ structures in 6 dimensions.

\section{Relating Hitchin's functionals in $6 \mathrm{D}$ to topological strings}

In the last section, we introduced two functionals $V_{H}(\rho), V_{S}(\sigma)$ which, when extremized, generate, respectively, a symplectic form $k$ and a closed holomorphic $(3,0)$ form $\Omega$ on a 6 -manifold $M$. This is reminiscent of the topological $\mathrm{A}$ and $\mathrm{B}$ models, and one might wonder whether there is some relation. In this section, we point out that such a relation does exist. Our arguments will be rigorous only at the classical level, but they suggest a natural extension to the quantum theories, which we will describe. One particularly interesting feature will emerge: namely, $V_{H}(\rho)$ turns out to be equivalent not to the $\mathrm{B}$ model itself but to a combination of the $\mathrm{B}$ and $\overline{\mathrm{B}}$ models.

\subsection{Hitchin's $V_{\mathrm{S}}$ as the A model}

We begin by discussing a relation between Hitchin's action functional (4.15),

$$
V_{S}(\sigma)=\int_{M} \sigma^{3 / 2}
$$

based on the closed 4-form $\sigma$, and the A model on $M$. As we discussed in Section 4.3, the solutions to the classical equations of motion coming from $V_{S}(\sigma)$ involve Kähler geometries, which are also the classical solutions of the A model Kähler gravity. In fact, more is true: the classical actions in both cases compute the volume of $M$. So at least at a superficial classical level, the two theories are equivalent. Moreover, we can argue that the small fluctuations in the two theories can be identified with one another. Namely, recall that in Hitchin's theory, we write $\sigma=\sigma_{0}+d \gamma$; then the action at quadratic order for the fluctuation $\gamma$ includes $\int d \gamma \wedge d^{c \dagger} \gamma$, which nicely matches the action for the quadratic fluctuations in the Kähler gravity theory. So one would expect that the two should be identified. 
Here we would like to take one more step in connecting the two theories: specifically, it has been recently argued [4] that the A model can be reformulated in terms of a topologically twisted $U(1)$ gauge theory on $M$, whose bosonic action contains the observables

$$
S=\frac{g_{s}}{3} \int_{M} F \wedge F \wedge F+\int_{M} k_{0} \wedge F \wedge F
$$

The partition function in this theory is a function of the fixed class $k_{0}$. The path integral can be defined as a sum over a gravitational "quantum foam" [4], i.e., over Kähler geometries with quantized Kähler class, ${ }^{4}$

$$
k=k_{0}+g_{s} F,
$$

or, equivalently, as a sum over ideal sheaves [46].

We claim that in the weak coupling $\left(g_{s} \rightarrow 0\right)$ limit, the theory based on the action (5.2) is equivalent to the "gravity theory" based on Hitchin's action (5.1). Moreover, we show that fixing the BRST symmetries of the Hitchin action naturally leads to the description of the A model as a topologically twisted supersymmetric $U(1)$ gauge theory.

In order to show this, we begin with the action

$$
S=\alpha \int_{M} \widetilde{F} \wedge \widetilde{F} \wedge \widetilde{F}-\beta \int_{M} \sigma \wedge \widetilde{F},
$$

where $\alpha$ and $\beta$ are some coefficients (which will be related to $g_{s}$ below), $\widetilde{F}$ is a 2 -form on $M$, and $\sigma$ is a 4 -form that varies in a fixed cohomology class,

$$
\begin{aligned}
{[\sigma] } & \in H^{4}(M) \quad \text { fixed, i.e., } \\
\sigma & =\sigma_{0}+d \gamma .
\end{aligned}
$$

At this point, we do not make any assumptions about the 2 -form $\widetilde{F}$; in particular, it need not be closed or co-closed.

First, let us integrate out the 2 -form $\widetilde{F}$ in the action (5.4). The equation of motion for $\widetilde{F}$ has the form

$$
3 \alpha \widetilde{F} \wedge \widetilde{F}-\beta \sigma=0 .
$$

This equation implies that the 2 -form $\widetilde{F}$ is a "square root" of $\sigma$, i.e., $\sigma$ is a stable 4 -form. Substituting $\widetilde{F}$ back into the action (5.4), we obtain precisely Hitchin's action (5.1), with the remaining path integral over a stable, closed 4-form $\sigma$. It is important to stress here that, since the action (5.4) is cubic in $\widetilde{F}$, the relation to Hitchin's action (5.1) holds only in the semi-classical

\footnotetext{
${ }^{4}$ We choose our normalization so that $F$ is integrally quantized: $\int_{C} F \in \mathbb{Z}$ for any closed 2-cycle $C \subset M$.
} 
limit. We return to this issue below, and show that this is precisely the limit $g_{s} \rightarrow 0$.

Similarly, starting with the action (5.4) and integrating out $\sigma$ one can obtain the $U(1)$ gauge theory (5.2). In order to see this, one has to eliminate $\sigma$ through its equations of motion, and then make a simple field redefinition. The equations of motion for $\sigma$ are very simple. Since the dynamical variable $\gamma$ appears as a Lagrange multiplier in equation (5.4), it leads to the constraint

$$
d \widetilde{F}=0,
$$

which means that the 2 -form $\widetilde{F}$ is closed and, therefore, can be interpreted as a curvature on a line bundle. The resulting action for $\widetilde{F}$ is

$$
S=\alpha \int_{M} \widetilde{F} \wedge \widetilde{F} \wedge \widetilde{F}-\beta \int_{M} \sigma_{0} \wedge \widetilde{F}
$$

In order to bring this action to the familiar form (5.2), it remains to do a simple change of variables. We introduce

$$
F=\widetilde{F}-\xi k_{0},
$$

where $\xi$ is a parameter and $k_{0}$ is the background Kähler form, such that $\sigma_{0}=k_{0} \wedge k_{0}$. Substituting equation (5.9) into equation (5.8), we get (up to a constant term) the action

$$
\begin{aligned}
S= & \alpha \int_{M} F \wedge F \wedge F+3 \xi \alpha \int_{M} F \wedge F \wedge k_{0} \\
& +\int_{M}\left(3 \xi^{2} \alpha k_{0} \wedge k_{0} \wedge F-\beta \sigma_{0} \wedge F\right) .
\end{aligned}
$$

Comparing equation (5.10) with equation (5.2) determines the parameters $\alpha, \beta$, and $\xi$ :

$$
\begin{gathered}
\alpha=\frac{g_{s}}{3}, \\
\xi=\frac{1}{g_{s}}, \\
\beta=\frac{1}{g_{s}} .
\end{gathered}
$$

With this choice of parameters, we find complete agreement between equation (5.10) and the $U(1)$ gauge theory action (5.2), including the numerical coefficients and the relation between the Kähler form $k$ and the field $F$. Indeed, substituting $\xi=1 / g_{s}$ into equation (5.9), we get

$$
\delta k=g_{s} F,
$$

which is precisely the required quantization condition (5.3). 
Summarizing, we find that equation (5.4) is equivalent to the gauge theory action (5.2) and, in the semi-classical limit, is also equal to Hitchin's action (5.1). In order to see when the semi-classical approximation is valid, it is convenient to write both terms in the action (5.4) with the same overall coefficient $1 / \hbar$. To achieve this, we rescale

$$
\widetilde{F} \rightarrow \gamma \widetilde{F},
$$

and set the coefficients in the two terms to be equal:

$$
\frac{1}{\hbar}=\alpha \gamma^{3}=\gamma
$$

In particular, the latter equality implies $\alpha=\frac{1}{\gamma^{2}}$. From the relations (5.11) and (5.14) it follows that the semi-classical limit, $\hbar \rightarrow 0$, corresponds to the limit $g_{s} \rightarrow 0$. Hence we conclude that the gauge theory action (5.2) is equivalent to Hitchin's action (5.1) precisely in the weak coupling limit.

\subsubsection{BRST symmetries and gauge fixing}

As noted before, we really want to connect Hitchin's theory to the topologically twisted version of the supersymmetric $U(1)$ gauge theory. In order to do this let us describe the BRST symmetries of the theory (5.4), which, as we just established, is equivalent to the $U(1)$ gauge theory (5.2). First, notice that the partition sum over the quantum foam can be viewed as a vacuum expectation value,

$$
\left\langle\exp \left(\frac{g_{s}}{3} \int \mathcal{O}_{1}+\int \mathcal{O}_{2}\right)\right\rangle
$$

in the topological $U(1)$ gauge theory on $M$, where $\mathcal{O}_{i}$ are the topological observables:

$$
\begin{aligned}
& \mathcal{O}_{1}=F \wedge F \wedge F, \\
& \mathcal{O}_{2}=k_{0} \wedge F \wedge F,
\end{aligned}
$$

Following [47], one can reconstruct the action of this topological 6-dimensional theory by studying the BRST symmetries that preserve equations (5.15) and (5.16). Writing (locally) $F$ as a curvature of a gauge connection $A$,

$$
F=d A,
$$

it is easy to see that equations (5.15) and (5.16) are invariant under the usual gauge transformations

$$
\delta A=d \epsilon_{0},
$$

as well as under more general transformations

$$
\delta A=\epsilon_{1}
$$


where the infinitesimal parameter $\epsilon_{1}$ is a 1 -form on $M$. The gauge fixing of the latter symmetry leads to a 1 -form ghost field $\psi$. Since $\epsilon_{1}$ itself has a gauge symmetry, $\epsilon_{1} \sim \epsilon_{1}+d \lambda$, one also has to introduce a commuting 0 form $\phi$ associated with this symmetry. Hence, already at this stage we see that the $6 D$ topological theory in question should contain a gauge field and a scalar. The only such theory is a maximally supersymmetric topological gauge theory in 6 dimensions, i.e., a theory with $\mathcal{N}_{T}=2$ topological supersymmetry. Equivalently, it is a theory with 16 real fermions, which can be identified with holomorphic $(p, 0)$-forms on $M$. Remember that on a Kähler manifold

$$
S(M) \cong \Omega^{0, *}(M) .
$$

The complete BRST multiplet in this theory looks like:

$$
\begin{aligned}
& \text { Bosons : } \quad 1-\text { form } A \\
& \text { cplx. scalar } \phi \\
& (3,0)-\text { form } \varphi \\
& \text { Fermions : } \quad \psi^{p, 0}, \psi^{0, p} \quad p=0,1,2,3
\end{aligned}
$$

Under the action of the BRST operator $s$, these fields transform as [48, 49]:

$$
\begin{array}{ll}
s \varphi^{0,3}=0 & s \varphi^{3,0}=\psi^{3,0} \\
s A^{0,1}=\psi^{0,1} & s A^{1,0}=0 \\
s \psi^{0,1}=0 & s \psi^{1,0}=-\partial_{A} \phi \\
s \psi^{0,0}=\left(k \cdot F^{1,1}\right) & s \psi^{2,0}=F^{2,0}
\end{array}
$$

This $\mathcal{N}_{T}=2$ 6-dimensional topological $U(1)$ gauge theory has been extensively studied in the literature, see e.g., [48-52]. A reduction of this theory on a Kähler 4-manifold $M^{4} \subset M$ leads to the $\mathcal{N}_{T}=4$ topological gauge theory studied in [53].

Finally, we identify the symmetry of Hitchin's action (5.1) that corresponds to the BRST symmetry (5.19). In order to do this, we need to find how this symmetry acts on the 4 -form field $\sigma$. Since in the $g_{s} \rightarrow 0$ limit the field $F$ is identified with the variation of the Käher form (5.12) it follows that

$$
\delta k=d \epsilon_{1},
$$

where $\sigma=k \wedge k$. It is easy to check that Hitchin's action (5.1) is indeed invariant under this symmetry,

$$
\delta S_{H}=\frac{3}{2} \int_{M} k \wedge \delta \sigma=3 \int_{M} k \wedge k \wedge \delta k=3 \int_{M} \sigma \wedge d \epsilon_{1}=0 .
$$

We have thus recovered the topologically twisted $U(1)$ theory which was conjectured in [4] to be equivalent to the quantum foam description of the A model. 


\subsection{Hitchin's $\mathrm{V}_{\mathrm{H}}$ as the $\mathrm{B}$ model}

Now we want to discuss the relation between Hitchin's "holomorphic volume" functional $V_{H}(\rho)$ and the B model (see also the recent work [54], which proposes a relation similar to what we will propose below). Classically, there is an obvious connection between the two, since solving the equations of motion of either one gives a closed holomorphic 3 -form $\Omega$. What about quantum mechanically? In order to understand this question, we must first recall a subtle feature of the $\mathrm{B}$ model partition function.

Consider the B model on a Calabi-Yau 3-fold $M$. This model is obtained by topological twisting of the physical theory with a fixed "background" complex structure, determined by a holomorphic 3 -form $\Omega$. The topological observables in this model are the marginal operators $\phi_{i}$ representing infinitesimal deformations of the complex structure, where $i=1, \ldots, h_{2,1}$. The partition function $Z_{B}$ was defined in [7] to be the generating functional of correlations of marginal operators: namely, introducing $h_{2,1}$ variables $x^{i}$, $Z_{B}\left(x, g_{s}, \Omega_{0}\right)$ obeys

$$
\left.D_{i_{1}} \cdots D_{i_{k}} Z_{B}\left(x, g_{s}\right)\right|_{x=0}=\left\langle\phi_{i_{1}} \cdots \phi_{i_{k}}\right\rangle_{\Omega_{0}} .
$$

More intrinsically, we can think of $x$ as labeling an infinitesimal deformation, so that for fixed $\Omega_{0}, Z_{B}\left(x, g_{s}, \Omega_{0}\right)$ is a function on the holomorphic tangent space $T_{\Omega_{0}} \mathcal{M}$ to the moduli space $\mathcal{M}$ of complex structures. By construction, this $Z_{B}$ is holomorphic in its dependence on $x$. But one gets one such function of $x$ for every $\Omega_{0}$, corresponding to all the different tangent spaces to $\mathcal{M}$, and one can ask how these different functions are related. This question was answered in [7], where the effect of an infinitesimal change in $\Omega_{0}$ was found to be given by a "holomorphic anomaly equation."

This $\Omega_{0}$ dependence of $Z_{B}$ was later reinterpreted in [8] as the wavefunction property. To understand what this means, it is convenient to combine $g_{s}$ and $x$ into a "large phase space" of dimension $h_{2,1}+1$. Changing $g_{s}$ is equivalent to an overall rescaling of $\Omega_{0}$ (which does not change the complex structure on $X$ ). So for fixed $\Omega_{0}$, we can consider $Z_{B}$ as a holomorphic function on $H^{3,0}(X, \mathbb{C}) \oplus H^{2,1}(X, \mathbb{C})$. Equivalently, $Z_{B}$ is a function on the "phase space" $H^{3}(X, \mathbb{R})$, which depends only on the complex combination of periods

$$
x_{I}=F_{I}-\tau_{I J}\left(\Omega_{0}\right) X^{J},
$$

and not on the conjugate combination

$$
\bar{x}_{I}=F_{I}-\bar{\tau}_{I J}\left(\Omega_{0}\right) X^{J} .
$$

This is similar to the idea of a wavefunction which depends only on $q$ but not on its conjugate variable $p$; indeed, $x_{I}$ and $\bar{x}_{I}$ are coordinates on $H^{3,0} \oplus$ 
$H^{2,1}$ and on $H^{1,2} \oplus H^{0,3}$ respectively, and they are indeed conjugate with respect to the standard symplectic structure on $H^{3}(X, \mathbb{R})$. Note that since $\tau$ depends on $\Omega_{0}$, changing $\Omega_{0}$ changes the symplectic coordinate system.

Now, if one is given a wavefunction $\psi(q)$ as a function of $q$ and one wants to express it as a function of $p$, there is a simple procedure for doing so: just take the Fourier transform. In fact, more generally, given $\psi(q)$ one can construct various different representations of the state, e.g., $\psi(p), \psi(p+q)$, $\psi(p+i q)$, and so on. Each such representation corresponds to a different choice of symplectic coordinates inside the $(p, q)$ phase space, and each can be obtained from $\psi(q)$ by an appropriate generalized Fourier transform. In [8] it was shown that the $\Omega_{0}$ dependence of $Z_{B}$ can be understood in exactly this way: starting from $Z_{B}\left(x, \Omega_{0}\right)$ one can obtain any other $Z_{B}\left(x, \Omega_{0}^{\prime}\right)$ by taking an appropriate Fourier transform! In this sense $Z_{B}$ is a wavefunction obtained by quantization of the symplectic phase space $H^{3}(X, \mathbb{R})$, which has various different representations depending on one's choice of symplectic coordinates for $H^{3}(X, \mathbb{R})$.

Now what about Hitchin's gravity theory? Consider the partition function $Z_{H}([\rho])$ of Hitchin's 6-dimensional gravity theory, formally written

$$
Z_{H}([\rho])=\int D \beta \exp \left(V_{H}(\rho+d \beta)\right)
$$

We do not expect that the formal expression (5.28) really captures the whole quantum theory, but the statement that $Z_{H}$ depends on a class $[\rho] \in$ $H^{3}(X, \mathbb{R})$ should be correct, as should the classical limit of equation (5.28). In comparing $Z_{H}$ to $Z_{B}$ we notice two points. First, unlike $Z_{B}, Z_{H}$ does not depend on a choice of symplectic coordinates for $H^{3}(X, \mathbb{R})$. Second, $Z_{H}$ depends on twice as many degrees of freedom as does $Z_{B}$ (because $Z_{B}$ depends on only half of the coordinates of $H^{3}(X, \mathbb{R})$ as explained above). So $Z_{H}$ cannot be equal to $Z_{B}$.

The situation changes drastically, however, if we combine the B model with the complex conjugate $\overline{\mathrm{B}}$ model. In that case, we have two wavefunctions, $Z_{B}$ and $\overline{Z_{B}}$, and we can consider the product state

$$
\Psi=Z_{B} \otimes \overline{Z_{B}} .
$$

(One could more generally consider a density matrix that is a sum of such pure product states). This product state sits inside a doubled Hilbert space, obtained from quantization of a phase-space which is also doubled, from $H^{3}(X, \mathbb{R})$ to $H^{3}(X, \mathbb{C})$. This doubled phase space has a polarization which does not depend on any arbitrary choice: namely, one can divide it into real and imaginary parts, and it is natural to ask for the representation of $\Psi$ as a function of the real parts of all the periods, $\Psi\left(\operatorname{Re} X_{I}, \operatorname{Re} F^{I}\right)$. This gives 
a function on $H^{3}(X, \mathbb{R})$ which does not depend on any choice of symplectic coordinates. This is actually a standard construction in quantum mechanics: the function one obtains expresses the density in phase-space corresponding to the wavefunction $Z_{B}$, and is known as the "Wigner function" of $Z_{B}$. It is this Wigner function which we propose to identify with $Z_{H}([\rho])$.

We can give an explicit formula for the Wigner function if we start from a particular representation of $Z_{B}$, namely the one corresponding to a basis of $\mathrm{A}$ and $\mathrm{B}$ cycles $\left\{A_{I}, B^{I}\right\}$ in $H_{3}(X, \mathbb{Z})$. (This choice of polarization corresponds to a certain limit in the space of possible $\Omega_{0}$; from now on we suppress $\Omega_{0}$ in the notation). Then $Z_{B}$ can be written as $Z_{B}\left(X_{I}\right)$, a function of the A cycle periods $X_{I}$, and we denote the B cycle periods $F^{I}$. Writing $P_{I}=\operatorname{Re} X_{I}$, $Q^{I}=\operatorname{Re} F^{I}$, the Wigner function is given by

$$
\Omega\left(P_{I}, Q^{I}\right)=\int d \Phi_{I} e^{-Q^{I} \Phi_{I}}\left|Z_{B}\left(P_{I}+i \Phi_{I}\right)\right|^{2} .
$$

This can be identified with $Z_{H}([\rho])$ if we identify $P_{I}$ and $Q^{I}$ as the (real) periods of the class $[\rho] \in H^{3}(X, \mathbb{R})$.

At least at string tree level (which in this context means large $\rho$ ) we can verify that this identification is correct. Namely, in that limit, $Z_{B}$ is dominated by the tree level free energy $F_{0}$, and writing $Z_{B}=e^{-\frac{i}{2} F_{0}}$, we can make a steepest descent approximation of the integral over $\Phi$ in equation (5.30). The argument of the exponential is

$$
-\frac{i}{2} F_{0}\left(P_{I}+i \Phi_{I}\right)+\frac{i}{2} \overline{F_{0}\left(P_{I}+i \Phi_{I}\right)}-Q^{I} \Phi_{I}
$$

The value of $\Phi$ which extremizes equation (5.31) occurs when $Q^{I}=$ Re $\partial F_{0} / \partial X_{I}=\operatorname{Re} F^{I}(P+i \Phi)$. At this $\Phi$, equation (5.31) becomes

$$
-\frac{i}{4} X_{I} F^{I}+\frac{i}{4} \overline{X_{I}} \overline{F^{I}}-\left(\operatorname{Re} F^{I}\right)\left(\operatorname{Im} X_{I}\right)=\frac{i}{4} X_{I} \overline{F^{I}}-\frac{i}{4} \overline{X_{I}} F^{I} .
$$

But this is exactly the classical Hitchin action $V_{H}=-\frac{i}{4} \int \Omega \wedge \bar{\Omega}$, evaluated at the value of $\Omega$ for which $\operatorname{Re} X_{I}=P_{I}$ and $\operatorname{Re} F^{I}=Q^{I}$. This establishes the desired agreement between $Z_{H}$ and the Wigner function of $Z_{B}$ at tree level. In fact, the above relation between the topological string and $\int \Omega \wedge \bar{\Omega}$ was already noted and used in [5], for the purpose of relating the topological string to $4 D$ black hole entropy. We will discuss this connection in Section 8.

It seems likely that the agreement between $Z_{H}$ and the Wigner function will also persist at one loop. The $\mathrm{B}$ model at one loop is known [7] to compute the Ray-Singer torsion of $M$, which is a ratio of determinants of $\bar{\partial}$ operators acting on forms of various degrees; these determinants should agree with those which appear in the quadratic expansion of $V_{H}$ around 
a critical point. This basically follows from the fact that the kinetic term is given by $\int \partial \phi \bar{\partial} \phi$, where $\phi$ is a $(1,1)$ form and the complex structure is determined by the choice of critical point. The difference from the $\mathrm{B}$ model is just that here $\phi$ is not viewed as a chiral field, so we get both the B and $\overline{\mathrm{B}}$ contributions; the one-loop contribution in the $\mathrm{B}$ model alone is a chiral determinant, the holomorphic square root of the determinant of the Laplacian.

Finally, we note that, by introducing an extra 3 -form field $H$, we can write the action functional $V_{H}(\rho)$ in a form that does not involve square roots, just as we did in equation (5.4) for the A model,

$$
S=\int_{M} \rho \wedge H+\int_{M} \alpha \cdot K_{a}^{b} K_{b}^{a}+\int_{M}(1-\alpha \cdot(\rho \wedge H)) \phi
$$

where $K_{a}{ }^{b}$ is defined in equation (4.27). It is easy to see that integrating out the 3 -form $H$ and the Lagrange multiplier $\phi$ leads to the holomorphic volume action $V_{H}(\rho)$ of equation (4.26). The action (5.33) could be useful for a "quantum foam" description of the B model parallel to the one discussed above for the A model.

\section{Reducing topological M-theory to form gravities}

In this section, we want to argue that the various form gravity theories we reviewed earlier arise naturally on supersymmetric cycles in topological M-theory. We will discuss various examples, but the basic idea is always the same: we consider a "local model" of a complete 7 -manifold $X$, obtained as the total space of an $m$-dimensional vector bundle over an $n$-dimensional supersymmetric (calibrated) cycle $M \subset X$, such that $m+n=7$,

$$
\begin{array}{ll}
\mathbb{R}^{m} \rightarrow & X \\
& \\
& \\
& \\
&
\end{array}
$$

This non-compact local model is intended to capture the dynamics of the 7-dimensional theory when the supersymmetric cycle $M$ shrinks inside a global compact $X$. This idea is natural when one recalls that the geometry of $X$ in the vicinity of a supersymmetric cycle $M$ is completely dictated by the data on $M$. Thus the local gravity modes induce a lower-dimensional gravity theory on $M$. This is similar to what is familiar in the context of string theory: near singularities of Calabi-Yau manifolds one gets an effective lower-dimensional theory of gravity. 
After making an appropriate ansatz, the 3 -form $\Phi$ on $X$ induces a collection of $p$-form fields on $M$; the equations of motion of topological M-theory,

$$
\begin{aligned}
d \Phi & =0, \\
d *_{\Phi} \Phi & =0,
\end{aligned}
$$

lead to equations of motion for the $p$-form fields on $M$. These equations of motion can be interpreted as coming from a topological gravity theory on $M$.

Depending on the dimension $n$ of $M$ and the vector bundle we choose over it, we will have various ansaetze for $\Phi$, leading to various gravity theories on $M$. For example, the cases $n=3$ and $n=4$ correspond, respectively, to associative and coassociative submanifolds, which are familiar examples of supersymmetric cycles in manifolds with $G_{2}$ holonomy. In these two cases, the corresponding vector bundle over $M$ is either the spin bundle over $M$ (when $M$ is associative) or the bundle of self-dual 2-forms over $M$ (when $M$ is coassociative). In order to obtain the other two gravity theories, namely the cases $n=2$ and $n=6$, one has to assume that the bundle (6.1) splits into a trivial line bundle over $M$ and a bundle of rank $m-1$. In this case, the holonomy group of $X$ is reduced to $S U(3)$, so that locally $X$ looks like a direct product,

$$
X=\mathbb{R} \times Y,
$$

where $Y$ is a Calabi-Yau 3-fold of the form (6.1), with $n+m=6$. Notice that apart from supersymmetric 2-cycles and 6-cycles, Calabi-Yau manifolds also contain supersymmetric 3-cycles and 4-cycles. A priori, the form gravity induced on the latter may be different from the gravity theory obtained on associative and coassociative cycles in a 7-manifold with the full holonomy $G_{2}$.

In the cases $n=3, m=4$ and $n=4, m=3$ we will be closely following two constructions of local $G_{2}$ manifolds given in $[55,56]$ and recently discussed in [45]. These two constructions have some common features which can be conveniently summarized in advance. We let $y^{i}$ denote a local coordinate on the fiber $\mathbb{R}^{m}$, and write $r=y_{i} y^{i}$. The ansatz is $S O(m)$ invariant, so that $\Phi$ depends only on $r$ and the coordinates on $M$. We construct a basis of 1 -forms in the fiber direction as

$$
\alpha^{i}=D_{A} y^{i}=d y^{i}+(A y)^{i}
$$

where $A$ is the 1 -form induced by a gauge connection on $M$ which acts on the $y^{i}$ in some representation. 
The fact that $\Phi$ is a stable 3 -form means that there exists a 7 -dimensional vielbein $e^{i}$ such that

$$
\Phi=e^{567}+e^{5} \wedge\left(e^{12}-e^{34}\right)+e^{6} \wedge\left(e^{13}-e^{42}\right)+e^{7} \wedge\left(e^{14}-e^{23}\right) .
$$

In the metric $g$ determined by $\Phi$, the $e^{i}$ form an orthonormal basis. We define a triplet of 2 -forms $\Sigma^{i}$ by the formula (3.14) as in the 2-form gravity:

$$
\begin{aligned}
& \Sigma^{1}=e^{12}-e^{34}, \\
& \Sigma^{2}=e^{13}-e^{42}, \\
& \Sigma^{3}=e^{14}-e^{23} .
\end{aligned}
$$

Then $\Phi$ is written

$$
\Phi=e^{567}+e^{i} \wedge \Sigma^{i}
$$

To verify the equations of motion, we will also need the expression for $*_{\Phi} \Phi$, derived straightforwardly by expanding in the $e^{i}$ :

$$
*_{\Phi} \Phi=-\frac{1}{6} \Sigma_{i} \wedge \Sigma^{i}+\frac{1}{2} \epsilon^{i j k} e^{i} \wedge e^{j} \wedge \Sigma^{k} .
$$

In fact, it is convenient to consider a slightly more general form of $\Phi$ : namely, rescaling the first three $e^{i}$ by $f$ and other four by $g$, we obtain

$$
\Phi=f^{3} e^{567}+f g^{2} e^{i} \wedge \Sigma^{i},
$$

and

$$
*_{\Phi} \Phi=-\frac{1}{6} g^{4} \Sigma_{i} \wedge \Sigma^{i}+\frac{1}{2} f^{2} g^{2} \epsilon^{i j k} e^{i} \wedge e^{j} \wedge \Sigma^{k}
$$

\subsection{D gravity on associative submanifolds}

One local model for a $G_{2}$ space $X$ is obtained by choosing $X$ to be the total space of the spin bundle over a 3 -manifold $M$. In this case, with our ansatz, the field content and equations of motion of topological M-theory on $X$ reduce to those of Chern-Simons gravity on $M$; in particular, the condition that $X$ has $G_{2}$ holonomy implies that $M$ has constant sectional curvature.

First, let us show that the field content of topological M-theory on $X$ can be naturally reduced to that of Chern-Simons gravity on $M$. This amounts to constructing an ansatz for the associative 3 -form $\Phi$ in terms of forms on $M$. We write it in the general form (6.8) and then impose the condition that $e^{1}, e^{2}, e^{3}, e^{4}$ are constructed out of an $S U(2)$ connection on $M$ acting on the spin bundle, as we explained earlier in equation (6.4): $e^{i}=\alpha^{i}, i=1, \ldots, 4$. 
For convenience we also relabel $e^{5}, e^{6}, e^{7}$ as $e^{1}, e^{2}, e^{3}$, so finally the form of $\Phi$ is

$$
\Phi=f^{3} e^{123}+f g^{2} e_{i} \wedge \Sigma^{i}
$$

where

$$
\begin{aligned}
& \Sigma^{1}=\alpha^{12}-\alpha^{34}, \\
& \Sigma^{2}=\alpha^{13}-\alpha^{42}, \\
& \Sigma^{3}=\alpha^{14}-\alpha^{23} .
\end{aligned}
$$

Further assume that $f, g$ depend only on the radial coordinate $r$, with $f(0)=$ $g(0)=1$. Then along $M$, the only fields (undetermined functions) in our ansatz are the dreibein $e^{i}$ and the $S U(2)$ connection $A^{i}$. These are exactly the fields of 3 -dimensional gravity in the first-order formalism, and they can be organized naturally into a complexified gauge field, as we discussed before.

Now we want to check that the equations of motion of topological M-theory reduce with our ansatz to those of 3-dimensional gravity. This amounts to evaluating $d \Phi$ and $d *_{\Phi} \Phi$ directly, using equation (6.9). One finds that if

$$
\begin{aligned}
& f(r)=\sqrt{3 \Lambda}(1+r)^{1 / 3}, \\
& g(r)=2(1+r)^{-1 / 6},
\end{aligned}
$$

then $d \Phi=0$ becomes equivalent to

$$
\begin{aligned}
d e & =-A \wedge e-e \wedge A, \\
d A & =-A \wedge A-\Lambda e \wedge e .
\end{aligned}
$$

The conditions (6.13) are precisely the equations of motion in 3D ChernSimons gravity,

$$
d \mathcal{A}+\mathcal{A} \wedge \mathcal{A}=0
$$

based on the gauge group $G$ as indicated in table 2. Furthermore, one can check that $d *_{\Phi} \Phi=0$ is automatic provided that, equation (6.12) and (6.13) are satisfied. So with this particular ansatz, the equations of motion of topological M-theory do indeed reduce to those of 3-dimensional gravity.

Table 2: Euclidean $3 D$ gravity can be viewed as a Chern-Simons theory.

\begin{tabular}{lccc}
\hline Cosmological constant & $\Lambda<0$ & $\Lambda=0$ & $\Lambda>0$ \\
\hline Gauge group $G$ & $S L(2, \mathbb{C})$ & $I S O(3)$ & $S U(2) \times S U(2)$ \\
\hline
\end{tabular}




\subsection{D gravity on coassociative submanifolds}

Another local model of a $G_{2}$ manifold is obtained by choosing $X$ to be the bundle of self-dual 2-forms over a 4-manifold $M$. We will see that in this case the effective gravity theory on $M$ is the 2 -form gravity we considered in Section 3.3.

First, let us show that the field content of topological M-theory on $X$ can be naturally reduced to that of 2 -form gravity on $M$. This amounts to constructing an ansatz for the associative 3 -form $\Phi$ in terms of forms on $M$. We write it in the general form (6.8) and then impose the condition that $e^{5}, e^{6}, e^{7}$ are constructed out of an $S U(2)$ connection on $M$ acting on the bundle of self-dual 2-forms, as we explained earlier: $e^{5}=\alpha_{1}, e^{6}=\alpha_{2}$, $e^{7}=\alpha_{3}$. Further assume that $\Sigma^{i}$ are purely tangent to $M$, and that $f, g$ depend only on the radial coordinate $r$, with $f(0)=g(0)=1$.

Thus along $M$ the associative 3 -form $\Phi$ can be simply written:

$$
\Phi=\alpha^{123}+\alpha^{1} \wedge \Sigma^{1}+\alpha^{2} \wedge \Sigma^{2}+\alpha^{3} \wedge \Sigma^{3} .
$$

Since we constructed both $\Phi$ and $\Sigma$ from the vielbein, which determines the metric, the metrics on $M$ which can be reconstructed from $\Phi$ and $\Sigma$ must agree. It is gratifying that this can be seen explicitly, as we now do: recall the expression for the $G_{2}$ metric in terms of $\Phi$ from equation (4.10),

$$
\sqrt{g} g_{j k}=-\frac{1}{144} \Phi_{j i_{1} i_{2}} \Phi_{k i_{3} i_{4}} \Phi_{i_{5} i_{6} i_{7}} \epsilon^{i_{1} \ldots i_{7}} .
$$

We wish to consider the components of the metric along $M, g_{j k}$, where $j, k=1, \ldots, 4$. Normalizing the normal directions to have length scale 1 , we can view $g$ in equation (6.16) as the determinant of the 4-dimensional metric on $M^{4}$. Also, notice that if $j, k=1, \ldots, 4$, then none of the $\Phi$-components can be $e^{567}$. Hence, all the components of $\Phi$ in equation (6.16) should contain $\Sigma^{i}, c f .(6.15)$.

Now, let us consider the combinatorial factors in equation (4.10). Since indices $j$ and $k$ are assumed to take values from 1 through 4 , one of the indices $i_{1}$ or $i_{2}$ in $\Phi_{j i_{1} i_{2}}$ can take values 5,6 , or 7 . Similarly, there are two choices to assign a normal direction to $i_{3}$ or $i_{4}$, and three choices in the last factor, $\Phi_{i_{5} i_{6} i_{7}}$. In total, we get a combinatorial factor $12=2 \cdot 2 \cdot 3$ and we can write the metric in the form

$$
\sqrt{g} g_{a b}=-\frac{1}{12} \Sigma_{a a_{1}}^{i} \Sigma_{b a_{2}}^{j} \Sigma_{a_{3} a_{4}}^{k} \epsilon^{i j k} \epsilon^{a_{1} a_{2} a_{3} a_{4}} .
$$

This is exactly the expression (3.17) for the metric on $M$ in the 2-form gravity. 
So we have written $\Phi$ in terms of an $S U(2)$ triplet of 2 -forms $\Sigma^{i}$, which by construction obey the constraint (3.13), and an $S U(2)$ gauge connection, which we used to define the $\alpha^{i}$. These are precisely the fields of the 2 -form gravity theory we considered above, which has self-dual Einstein metrics on $M$ as its classical solutions. Note that from the viewpoint of the 4dimensional form theory of gravity, the $S U(2)$ gauge indices $i=1,2,3$ and the 4-dimensional space-time indices of $\Sigma_{a b}^{i}$ are unrelated. However, we have seen that in the context of topological M-theory the $3 S U(2)$ gauge indices are unified with the 4 space-time indices to give a 3-form in seven dimensions. This by itself is rather satisfactory, and suggestive of a deep role for topological M-theory in the context of 4-dimensional quantum gravity.

Next we want to argue that the field equations of topological M-theory reduce to those of the 2 -form gravity theory on $M$. First consider the equation $d \Phi=0$. A direct computation shows that with the choice

$$
\begin{aligned}
& f(r)=(1+r)^{-1 / 4}, \\
& g(r)=\sqrt{2 \Lambda}(1+r)^{1 / 4},
\end{aligned}
$$

the condition $d \Phi=0$ becomes equivalent to

$$
\begin{gathered}
D_{A} \Sigma=0, \\
F \wedge \Sigma=0 .
\end{gathered}
$$

In fact, the latter equation follows from the former by applying $D_{A}$ to both sides; so we just have to impose $D_{A} \Sigma=0$, which means that $A$ is the $S U(2)_{+}$ part of the spin connection. Note that this also implies that $F$ is self-dual in the metric induced by $\Sigma, F=F_{+}$; this follows from the fact that $F$ is $S U(2)_{+}$valued, and the symmetry $R_{a b c d}=R_{c d a b}$ of the Riemann tensor, which is shared by $F$.

Similarly, one finds that $d *_{\Phi} \Phi=0$ can be satisfied provided that

$$
F_{+}=\frac{\Lambda}{12} \Sigma \text {. }
$$

So altogether, the equations of motion of topological M-theory imply

$$
\begin{aligned}
& D_{A} \Sigma=0, \\
& F=\frac{\Lambda}{12} \Sigma .
\end{aligned}
$$

These agree precisely with equation (3.21). In sum, the field content and equations of motion of the self-dual version of 2 -form gravity agree with those of topological M-theory, when we make a special ansatz for $\Phi$. 


\section{3 $6 \mathrm{D}$ topological strings}

Finally let us consider the case $n=6$. In this case $X$ is a real line bundle over the 6-dimensional $M$, and we choose it to be trivial - either $X=M \times \mathbb{R}$ or its compactification $X=M \times \mathbf{S}^{1}$. Let $\mathbb{R}$ be parameterized by $t$. Then a natural ansatz for $\Phi$ is

$$
\Phi=\rho+k \wedge d t
$$

where $\rho$ and $k$ are, respectively, a 3 -form and 2 -form on $M$. If $\Phi$ is a stable 3 -form on $X$, then $\rho$ and $k$ are stable on $X$, so as we discussed earlier, they define respectively an almost complex structure and a presymplectic structure on $X$, and if we impose also the conditions (4.30) and (4.31) then these two structures are compatible. In that case they give an $S U(3)$ structure on $X$. The condition that this $S U(3)$ structure is integrable,

$$
\begin{aligned}
& d k=0, \\
& d(\rho+i \widehat{\rho})=0,
\end{aligned}
$$

is equivalent to the 7-dimensional equations of motion $d \Phi=0, d *_{\Phi} \Phi=0$. So with this ansatz, topological M-theory on $X$ reduces to a theory on $M$, describing variations of $k$ and $\rho$, for which the classical solutions are CalabiYau 3-folds.

What 6-dimensional theory is this? As they are usually conceived, neither the topological A model nor the topological B model alone fits the bill: at least perturbatively, the A model just describes variations of $k$, and the $\mathrm{B}$ model those of the holomorphic 3-form $\Omega=\rho+i \widehat{\rho}$. The theory we are getting on $M$ is a combination of the A and B models - with a slight coupling between them, expressed by the constraints (4.30) and (4.31). In support of this point of view, note that after imposing (4.30) and (4.31), the action $V_{7}(\Phi)$ can be simply expressed in terms of $\rho$ and $k$ : it becomes simply

$$
V_{7}(\Phi)=3 V_{S}(k)+2 V_{H}(\rho)
$$

where $V_{S}$ and $V_{H}$ are the 6-dimensional symplectic and holomorphic volume functionals introduced in Section 4.3. As we discussed in Section 5, these functionals correspond respectively, to the $\mathrm{A}$ model and the $\mathrm{B}+\overline{\mathrm{B}}$ model.

It is natural to conjecture that this 7-dimensional construction is in fact related to the non-perturbative completion of the topological string, which we expect to mix the A and B models, and to related phenomena such as the topological S-duality conjectured in $[1,2]$ (see also [57]). While this picture is far from complete, there is one encouraging sign, which we will describe further in the next section. 


\section{Canonical quantization of topological M-theory and S-duality}

In the last section we discussed a possible relation between topological M-theory on $X=M \times \mathbf{S}^{1}$ and topological string theory on $M$. In particular, we found that the classical reduction, obtained just by considering fields which are independent of the coordinate along $\mathbf{S}^{1}$, leads to a combination of two systems, which are in the universality classes of the topological A and B models. Recently, there have been some hints that the A and B model could be coupled to one another. In this section, we discuss how such a coupling could arise through canonical quantization, and how this relates to the notion that the topological string partition function is a wave function.

We begin by considering the 4-form version of topological M-theory. To perform the canonical quantization of Hitchin's action $V_{7}(G)$ is a non-trivial problem, because of the usual subtleties involved in quantizing a diffeomorphism invariant theory. Moreover, we should note that we are viewing Hitchin's action only as an effective action, which we are using just to extract some basic facts about the Hilbert space. For this purpose it is enough to work roughly, although a more precise treatment would certainly be desirable.

So let us consider the 7-dimensional gravity theory (4.39) on a manifold $X=M \times \mathbb{R}$, where $M$ is a compact 6 -manifold and $\mathbb{R}$ is the "time" direction. We decompose the 3 -form gauge field $\Gamma$ as

$$
\Gamma=\gamma+\beta \wedge d t
$$

where $\gamma$ and $\beta$ are a 3 -form and 2-form, respectively, with components only along $M$. Similarly decompose $G$ and ${ }^{*} G$ as

$$
\begin{aligned}
G & =\sigma+\widehat{\rho} \wedge d t, \\
*_{G} G & =\rho+k \wedge d t .
\end{aligned}
$$

Then write

$$
\begin{aligned}
G & =G_{0}+d \Gamma \\
& =\left(\sigma_{0}+d \gamma\right)+\left(\widehat{\rho}_{0}+d \beta+\dot{\gamma}\right) \wedge d t,
\end{aligned}
$$

so that

$$
\begin{aligned}
& \widehat{\rho}=\widehat{\rho}_{0}+d \beta+\dot{\gamma}, \\
& \sigma=\sigma_{0}+d \gamma .
\end{aligned}
$$

The configuration space is spanned by the components $(\gamma, \beta)$ of the gauge field $\Gamma$. Their conjugate momenta are

$$
\pi_{\gamma}=\frac{7}{4} \rho, \quad \pi_{\beta}=0 .
$$


The longitudinal component $\beta$ is an auxiliary field; it imposes the constraint

$$
\frac{\delta V_{7}}{\delta \beta}=d \rho=0,
$$

which generates the spacelike, time-independent gauge transformations $\gamma \rightarrow \gamma+d \lambda$. Hence the reduced phase-space which we obtain from canonical quantization of the 4 -form theory is parameterized by $(\gamma, \rho)$, where

$$
\gamma \in \Omega^{3}(M) / \Omega_{\text {exact }}^{3}(M), \quad \rho \in \Omega_{\text {closed }}^{3}(M) .
$$

Now let us compute the Hamiltonian. Suppose that we impose the conditions (4.30) and (4.31) (we will comment more on the role of these constraints later.) Then it is straightforward to verify that $\rho$ and $\widehat{\rho}$ are related as in Section 4.3, and $\sigma=\frac{1}{2} k \wedge k$. The action $V_{7}$ from equation (4.39) becomes

$$
V_{7}=\int_{X} d t\left(2 V_{H}(\rho)+3 V_{S}(\sigma(\gamma))\right)
$$

Using equation (7.4) we can construct the Hamiltonian,

$$
H=2 V_{H}(\rho)+3 V_{S}(\sigma(\gamma))-\dot{\gamma} \wedge \pi_{\gamma}=\frac{3}{2}\left(2 V_{S}(\sigma(\gamma))-V_{H}(\rho)\right)
$$

Although our treatment has been rough, we can gain some confidence from the fact that the Hamiltonian we ultimately obtained at least gives classical equations of motion agreeing with the Lagrangian formulation; namely, it agrees with equation (4.47), which indeed defines a flow giving $G_{2}$ holonomy metrics. A more precise treatment (possibly starting from a different classically equivalent action) would require a better understanding of the constraints (4.30) and (4.31); we believe that they will turn out to be the diffeomorphism and Hamiltonian constraints, as usual in diffeomorphism invariant theories. Indeed, note that (4.31) is simply the constraint $H=0$.

As usual in the Hamiltonian formalism, we treat $\rho, \gamma$ as the canonical variables, where $\rho$ is "momentum" and $\gamma$ is "position." From equation (7.4) we see that they have canonical commutation relations

$$
\{\delta \gamma, \delta \rho\}=\int_{M} \delta \gamma \wedge \delta \rho
$$

Recalling that $V_{H}$ and $V_{S}$ were identified, respectively, with the $\mathrm{B}$ and $\mathrm{A}$ models, we see that the Hamiltonian has split into a "kinetic term" involving the B model and a "potential term" involving the A model. Despite this splitting, the A and B models are not independent; the fact that $\rho$ and $\gamma$ do not commute at the same point of $M$ suggests that, for the quantum Calabi-Yau, the uncertainty principle would prevent measurements of the complex structure and Kähler structure at a given point from being done simultaneously. This is an interesting result which deserves more scrutiny. 
One can also ask about the commutation relations between the zero modes of the A and B model fields, which might be of more direct interest, because these zero modes are observables on which the partition function depends. Some of these zero modes are already present in the heuristic construction of the phase-space which we gave above. For example, one can consider variations of $\gamma$ which are closed, $d(\delta \gamma)=0$; these induce no variation in the Kähler form, but nevertheless affect the non-perturbative A model partition function (via the coupling to Lagrangian branes) as we discussed in Section 3. These variations of $\gamma$ up to gauge equivalence parameterize an $H^{3}(M, \mathbb{R})$ in the phase-space, which via equation (7.9) is canonically conjugate to the $H^{3}(M, \mathbb{R})$ given by the cohomology class of $\rho$. This means that the A model variables and $\mathrm{B}$ model variables mix; the parameter playing the role of the Lagrangian D-brane tension in the A model is conjugate to the 3 -form of the B model.

There is a dual version of the above discussion: if we had started from the 3-form version of topological M-theory instead of the 4-form version, we would have written

$$
\rho=\rho_{0}+d B,
$$

where $B$ is a 2 -form on $M$. (This field is very closely related to the field that we denoted as $\phi$ that appeared in the B model topological string.) The phase-space then turns out to be spanned by $B$ and $\sigma$ with the Poisson bracket given by

$$
\{\delta B, \delta \sigma\}=\int_{M} \delta B \wedge \delta \sigma .
$$

This pairing agrees with the one we obtained above, except that it includes different zero modes: instead of having two copies of $H^{3}(M, \mathbb{R})$ we now have the variations of $B$ up to gauge equivalence which do not change $\rho$, parameterizing an $H^{2}(M, \mathbb{R})$, canonically conjugate to the $H^{4}(M, \mathbb{R})$ given by the cohomology class of $\sigma$. Hence, the $B$-field which couples to the D1brane of the $\mathrm{B}$ model is conjugate to the Kähler parameter of the A model.

In sum, we seem to be finding that even at the level of the zero modes, i.e., the observables, there is a sense in which the fields of the A and B models are conjugate to one another. It is natural to suspect that this is related to the conjectured S-duality between the A and B models, which would be interpreted as position/momentum exchange or electric/magnetic duality in topological M-theory. In particular, the fact that non-perturbative amplitudes of the B model involve the D1-brane and the $B$-field, and the fact that the non-perturbative amplitudes of the A model involve Lagrangian D-branes and the $\gamma$ field, suggest that the full non-perturbative topological string is a single entity consisting of the A and B models together. 
Clearly these ideas should be developed further, but we feel that there is a beautiful connection here, between the conjectured S-duality between the $\mathrm{A}$ and $\mathrm{B}$ models and the fact that topological M-theory treats their degrees of freedom as conjugate variables.

One might ask how this Hamiltonian quantization is related to the fact that the B model partition function is a wavefunction, reviewed in Section 5.2, which was one of our original motivations for introducing a 7dimensional topological M-theory. In the zero mode sector we have found two conjugate copies of $H^{3}(X, \mathbb{R})$, which would be sufficient to account for both the phase-spaces underlying the $\mathrm{B}$ model partition function and the $\overline{\mathrm{B}}$ model partition function. This is reminiscent of Section 5.2 where we saw that we could interpret the Wigner function as a wavefunction for the combined $\mathrm{B}$ and $\overline{\mathrm{B}}$ models, with the zero mode phase-space $H^{3}(X, \mathbb{C})$, parameterized by the conjugate variables $\operatorname{Re} \Omega=\rho$ and $\operatorname{Im} \Omega=\widehat{\rho}$. On the other hand, as dicussed above, in the 7-dimensional theory $\gamma$ is conjugate to $\rho$. We are thus naturally led to identify $\widehat{\rho}=\gamma$. This identification was in a sense predicted by the topological S-duality conjecture, since it says precisely that the Lagrangian D-branes of the A model are coupled to the imaginary part of $\Omega$. It indeed follows semi-classically if we identify the wavefunction as being given by the Hitchin functional, $\Psi(\rho) \sim \exp V_{H}(\rho)$; then we get the necessary relation

$$
\gamma|\Psi\rangle=\widehat{\rho}(\rho)|\Psi\rangle
$$

using $\delta V_{H} / \delta \rho=\widehat{\rho}$. This relation between the potential and the wavefunction is not unexpected, since the function $V_{H}$ is quadratic in $\rho$.

Note that we expect to recover the Wigner function (5.30) by considering topological M-theory on a 7 -manifold $X=M \times \mathbf{S}^{1}$, where $M$ is a CalabiYau space. This is natural to expect from dimensional reduction, making contact with the discussion of Section 5.2.

\section{Form theories of gravity and the black hole attractor mechanism}

In the previous sections we have discussed various theories of gravity in which one reconstructs geometric structures from $p$-forms on the spacetime $M$. Although this might seem like an unusual way to get these structures, a similar phenomenon occurs in superstring theory compactified on $M$ : given a black hole charge, which can be represented as an integral cohomology class on $M$, the attractor mechanism fixes certain metric data $g_{\mu \nu}$ of $M$ at 
the black hole horizon [58-61]. In other words, it provides a map ${ }^{5}$

$$
Q \mapsto g_{\mu \nu}
$$

In this section, we will discuss a relation between black holes and Hitchin's functionals. In particular, we argue that these functionals also lead to the map (8.1). In a sense, the metric flow of the internal manifold from spatial infinity to the black hole horizon can be viewed as a geodesic flow with respect to Hitchin's action. In fact, Hitchin's picture is more general: it does not assume the metric to be of the Calabi-Yau form, but derives that from the same action principle which leads to the relation between the charge and metric. The usual attractor mechanism only discusses the zero mode sector of the metric, whereas Hitchin's action also deals with the massive modes.

This link between form theories of gravity and BPS black holes can, in fact, lead to a fundamental non-perturbative definition of the gravitational form theory as counting black hole degeneracies with a fixed charge, as in the recent work $[5,6]$. This interpretation of the gravitational form theories also "explains" why one fixes the cohomology class of the form and integrates only over massive modes; this corresponds simply to fixing the black hole charge. At least in the cases of $4 D$ and $5 D$ BPS black holes, we will show that this interpretation is correct at leading order in the black hole charge; this amounts to the statement that the value of the extremized classical action agrees with the semi-classical black hole entropy.

\subsection{BPS black holes in 4 dimensions}

We begin with the case of $4 D$ BPS black holes in Type IIB string theory compactified on a Calabi-Yau 3-fold $M$. In Section 4.3 we defined Hitchin's "holomorphic volume" (4.25), a functional of a 3-form $\rho$ in 6 dimensions:

$$
V_{H}(\rho)=\frac{1}{2} \int_{M} \widehat{\rho} \wedge \rho=-\frac{i}{4} \int_{M} \Omega \wedge \bar{\Omega} .
$$

Furthermore, we noted that, if we hold the cohomology class $[\rho]$ fixed (writing $\left.\rho=\rho_{0}+d \beta\right)$, the critical points of $V_{H}(\rho)$ yield holomorphic 3 -forms on $M$ with real part $\rho$. So the process of minimizing $V_{H}$ produces the imaginary part of $\Omega$ as a function of its real part. Remarkably, this is exactly what the attractor mechanism does: fixing the black hole charge $C$ for the theory on $\mathbb{R}^{4}$, the attractor mechanism produces the value of $\Omega$ of the Calabi-Yau at

\footnotetext{
${ }^{5}$ More precisely, it fixes some of the components of $g$; not all of the moduli are fixed by the attractor mechanism.
} 
the black hole horizon, and the real part of $\Omega$ is equal to $C^{*}$, the Poincare dual of $C$. Therefore, it is natural to identify

$$
[\rho]=C^{*} .
$$

Note that the quantization of $C$ matches the fact that $\rho$ is naturally quantized, if we view it as the field strength of the 2 -form potential $\beta$. So the holomorphic volume functional $V_{H}$ is related to the attractor mechanism at least classically.

Furthermore, the classical value of the action also has a natural physical meaning: namely, after fixing $C$, the value of $\int \Omega \wedge \bar{\Omega}$ at the critical point gives the leading-order contribution to the black hole entropy at large $C$. Now consider the quantum theory with action $V_{H}$. The path integral formally defines a partition function $Z_{H}(C)$ depending on the charge,

$$
Z_{H}(C)=\int_{[\rho]=C^{*}} D \rho \exp \left(V_{H}(\rho)\right) .
$$

We conjecture that this path integral computes the exact number of states of the black hole (or more precisely the index $Z_{B H}(C)$ defined in [5], which counts the states with signs):

$$
Z_{B H}(C)=Z_{H}(C)
$$

The main evidence for this conjecture is that if the path integral (8.4) exists, it would be a function of $C$ whose leading asymptotics agree with the black hole entropy - it would be remarkable if there were two such functions with natural physical definitions and they were not equal. Conversely, one could define the non-perturbative quantum theory by the black hole entropy.

Additional evidence for the conjecture (8.5) comes by noticing that it is essentially the conjecture of [5], which identified $Z_{B H}(C)$ with a Wigner function constructed from the B model partition function $Z_{B}$. Namely, choose a splitting of $H^{3}(M, \mathbb{Z})$ into $\mathrm{A}$ and $\mathrm{B}$ cycles. Then splitting $C$ into electric and magnetic charges, $C=(P, Q)$, one has [5]

$$
Z_{B H}(C)=\int d \Phi e^{i Q^{I} \Phi_{I}}\left|Z_{B}(P+i \Phi)\right|^{2} .
$$

On the other hand, as we already discussed in Section 5.2, there is indeed a relation (5.30) between the B model and Hitchin's theory,

$$
Z_{H}(C)=\int d \Phi e^{i Q^{I} \Phi_{I}}\left|Z_{B}(P+i \Phi)\right|^{2}
$$

Recall that Hitchin's theory based on $V_{H}$ is related not to the B model but to the $\mathrm{B}$ plus $\overline{\mathrm{B}}$ model; this agrees well with the fact that this $\mathrm{B}$ plus $\overline{\mathrm{B}}$ model also appears in the counting of black hole entropy. This makes one 
more confident that the connection between Hitchin's theory and the black hole is direct and deep.

\subsection{BPS black holes in 5 dimensions}

So far we have discussed a relation between $V_{H}$ and counting of 4-dimensional BPS black hole states obtained from Type II string theory on $M$. But as described in Section 4.3, there is also the functional $V_{S}$ which makes sense on the 6-manifold $M$; one could ask whether it is also related to black hole entropy. In this section, we will argue that it is, and the black holes in question are the ones in the five-dimensional theory obtained by compactifying M-theory on $M$. These BPS black holes can be constructed by wrapping M2-branes over 2-cycles of $M$, and are characterized by a charge $Q \in H_{2}(M, \mathbb{Z})=H^{4}(M, \mathbb{Z})$ and a spin $j$. At first, let us set $j=0$. To connect the black hole counting to Hitchin's theory based on $V_{S}$, we identify

$$
Q^{*}=[\sigma] \text {. }
$$

The attractor value of the moduli in this case is given [61, 62] by a Kähler form $k$, such that $\frac{1}{2} k^{2}=\sigma$; with this value of $k$, the volume of the CalabiYau is proportional to the entropy of the black hole,

$$
S_{B H} \sim \int_{M} k^{3}=\int_{M} \sigma^{3 / 2} .
$$

In other words, the black hole entropy is given by the classical value of $V_{S}(\sigma)$. This is automatically consistent with the fact that the black hole entropy in five dimensions scales as $Q^{3 / 2}$. So, in parallel with what we did for $V_{H}$, we conjecture that the partition function $Z_{S}([\sigma])$ of the theory based on $V_{S}$ counts BPS states of five-dimensional black holes.

It is possible to extend the foregoing discussion to spinning black holes, by introducing an additional 6 -form field $J$ in the Hitchin action $V_{S}$. We denote the integral cohomology class of $J$ by $j=[J] \in H^{6}(M, \mathbb{Z})$; this $j$ can be naturally identified with the spin of the black hole. We consider the action

$$
V_{S}(\sigma, J)=\int \sqrt{\sigma^{3}-J^{2}}
$$

where

$$
\begin{aligned}
\sigma^{3}-J^{2}= & \left(\sigma_{i_{1} i_{2} i_{3} i_{4}} \sigma_{j_{1} j_{2} j_{3} j_{4}} \sigma_{k_{1} k_{2} k_{3} k_{4}}\right. \\
& \left.-J_{i_{1} i_{2} j_{1} j_{2} k_{1} k_{2}} J_{i_{3} i_{4} j_{3} j_{4} k_{3} k_{4}}\right) \epsilon^{i_{1} i_{2} j_{1} j_{2} k_{1} k_{2}} \epsilon^{i_{3} i_{4} j_{3} j_{4} k_{3} k_{4}} .
\end{aligned}
$$

It is easy to see that this modification does not change the attractor value of the Kähler form $k$, but changes the classical value of the action to $\sqrt{Q^{3}-j^{2}}$, which agrees with the entropy of the spinning black hole. 
We have just argued that the quantum theory based on the extended functional (8.10) should count the degeneracies of BPS black holes in five dimensions. On the other hand, since the perturbative A model counts exactly these degeneracies [63], one might expect a direct relation between the A model and equation (8.10). At least for $j=0$, we have already encountered this relation in Section 5.1, where the quantum foam description of the A model was related to a Polyakov version of $V_{S}$.

\subsection{Other cases}

It is natural to conjecture that the relation between BPS objects and form gravity theories goes beyond the examples discussed above. In particular, it would be interesting to develop this story for the case of $G_{2}$ manifolds. For example, in M-theory compactified on a $G_{2}$ manifold, we can consider BPS domain walls formed from M5-branes wrapped on associative 3-cycles. It is natural to conjecture that the quantum version of Hitchin's 4-form theory is computing the degeneracies of these domain walls. In the Type IIB superstring compactified on a $G_{2}$ manifold, one can similarly ask about the degeneracy of BPS strings obtained by wrapping D5-branes on coassociative 4-cycles; one might expect a relation between this counting and the quantum version of Hitchin's 3-form theory.

\section{Topological $\mathrm{G}_{2}$, twistors, holography, and $4 \mathrm{D}$ gauge theories}

In this section, we discuss possible dualities relating three different theories:

i) gauge theory on a Riemannian 4-manifold $M$;

ii) topological A model on the twistor space, $T(M)$, of a 4-manifold $M$;

iii) topological M-theory on a 7 -manifold $X$,

$$
\begin{array}{ll}
\mathbb{R}^{3} \rightarrow & X \\
& \downarrow \\
& M
\end{array}
$$

As we reviewed earlier, the 7 -manifold $X$ admits a natural metric with $G_{2}$ holonomy if $M$ is a self-dual (i.e., with self-dual Weyl tensor) Einstein 4-manifold. ${ }^{6}$ In that case, the $\mathbb{R}^{3}$ bundle (9.1) is the bundle of self-dual

\footnotetext{
${ }^{6}$ Such manifolds are also known as quaternionic Kähler manifolds of dimension one.
} 
2-forms on $M$. Let us compare this to the corresponding geometric structure on the twistor space $T(M)$.

First, let us recall the definition of the twistor space $T(M)$. Consider the space of self-dual 2-forms of norm 1 . For each point on $M$ this gives rise to a 2 -sphere. The total space is the twistor space, $T(M)$, which has a canonical almost complex structure and also a canonical map to $M$, with fiber being the twistor sphere $\mathbf{C P}^{1}$. There is a remarkable connection between self-dual metrics on $M$ (not necessarily Einstein) and the integrability of the almost complex structure on $T(M): T(M)$ has an integrable complex structure if and only if $M$ is self-dual $[64,65]$. Moreover, $T(M)$ admits a Kähler structure if and only if $M$ is Einstein [38] (see also [66]). These are the necessary conditions for the existence of topological A and B models on $T(M)$.

In order to complete this to a string theory we also need conformal invariance, which is usually guaranteed by a Ricci-flatness condition. This is not the case, however, for the twistor space $T(M)$, which is not Ricci-flat. One can complete $T(M)$ to a Ricci-flat supermanifold by including extra fermionic directions [67]. We want to explore another way of obtaining Ricci-flatness. As discussed above, the bundle $X$ of self-dual 2-forms over $M$ has a natural $G_{2}$ holonomy metric, so in particular it is Ricci-flat. On the other hand, the boundary of $X$ is precisely the twistor space,

$$
T(M)=\partial X
$$

In this sense, we could view $X$ as obtained by adjoining a radial direction to $T(M)$.

So we could define a topological string theory on the twistor space $T(M)$ as a holographic dual to topological M-theory on $X$. We note that the A model can be defined on 3-folds which are not necessarily Calabi-Yau; it has been studied in the mathematical literature on Gromov-Witten theory [6871]. Conversely, using the Gromov-Witten theory on $T(M)$ we can define topological M-theory on $X$, at least perturbatively.

This holographic duality is reminiscent of our original motivation to look for a 7-dimensional theory, which would naturally explain the observation that topological string partition function should be viewed as a wavefunction. We also note that, in the present case, the boundary 6-manifold is not stationary under Hitchin's Hamiltonian flow equations; this reflects the fact that $T(M)$ is not a Calabi-Yau. 


\subsection{Large N holography and gravitational holography}

We are familiar with examples of holography in the context of open-closed string dualities, where in the large $N$ limit D-branes wrapping some cycles disappear and the theory is best described by a new geometry obtained by deleting the locus of the D-branes, replaced by suitable fluxes. Via this holographic duality, the open string gauge theory provides an answer to questions of gravity in a geometry obtained by a large $N$ transition.

Another kind of duality — which is somewhat similar to holography — is a duality between M-theory on an interval and the heterotic $E_{8} \times E_{8}$ theory living on the boundary [72]. In this duality, the coupling constant of the heterotic string controls the size of the interval. Even though the heterotic string "lives" on the boundary, it can be used, at least in principle, to study gravitational physics in the bulk.

The relation between the 7-dimensional topological M-theory on $X$ and the topological string on a 6-manifold $T(M)$ is more similar to the heterotic/M-theory duality. In this sense, when we say that the partition function of a topological string theory on the twistor space can be regarded as a wave function in a 7 -dimensional theory on $X$, what we mean is a "gravity/gravity holography."

Having said that, it is natural to ask: is there an open-closed string holographic duality in the present context? Given that we do not yet have a deep understanding of topological M-theory, we will limit ourselves to some string-motivated speculations below.

In order to have an open/closed duality, we need to be working in some context where D-branes exist. From the point of view of embedding of the $G_{2}$ theory in the physical M-theory, it is natural to compactify on one more circle and obtain a Type IIA string theory compactification on a $G_{2}$ manifold. So let us consider Type IIA on a non-compact $G_{2}$ manifold $X$ of the form (9.1), with $N$ D-branes wrapped over the coassociative 4-manifold $M$ (in the full superstring theory these could be viewed for example as spacetime-filling D6-branes). By analogy with geometric transitions in CalabiYau tree-folds [73], in the large $N$ limit we expect a transition to a new geometry which can be obtained by removing the locus of the D-branes,

$$
X \backslash M \text {. }
$$

This space is a real line bundle over $T(M)$. From the discussion earlier in this section, we expect topological M-theory on this 7-manifold to be related to topological string theory on $T(M)$. This leads to a natural conjecture that topological gauge theory on a 4 -manifold $M$ is a holographic dual to 
topological string theory on the twistor space $T(M)$. This dovetails in a natural way with the idea that the topological string partition function should be viewed as a wave function on the boundary of the 7-dimensional manifold with $G_{2}$ holonomy.

What kind of topological gauge theory in four dimensions should we expect? The most natural conjecture is that it is the self-dual Yang-Mills theory, which is related to the D-brane theory for $\mathcal{N}=2$ strings. In other words, one might conjecture that the self-dual Yang-Mills on $M$ is dual to topological strings on $T(M)$, so that the Kähler class of $\mathbf{C P}^{1} \in T(M)$ is identified with the ' $\mathrm{t}$ Hooft parameter of the dual gauge theory, $t=N g_{s}$. Below, we consider this duality in more detail for $M=\mathbf{S}^{4}$ and $T(M)=\mathbf{C P}^{3}$.

Topological string theory on $T(M)=\mathbf{C P}^{3}$ is rather trivial due to the $U(1)$ charge conservation on the worldsheet. In particular, the free energy is simply given by the cubic classical triple intersection of $\mathbf{C P}^{3}$. This agrees with the fact that self-dual Yang-Mills is also trivial in perturbation theory. However, it is known that topological strings can be made more interesting by turning on higher charge $(q, q)$ form operators with $q=2,3$. The most natural one is the volume form with $q=3$, which preserves all the symmetries of $\mathbf{C P}^{3}$. Once we add an operator $s \Phi_{3,3}$, the topological A model string on $\mathbf{C P}^{3}$ becomes non-trivial and receives all order corrections. Thus, the partition function of the perturbed A model is a function of two independent variables,

$$
Z^{\text {top }}\left(g_{s}, s^{2} e^{-t}\right) \text {. }
$$

The fact that the combination $s^{2} e^{-t}$ appears follows from charge conservation of the topological A model.

One possibility is to look for a deformation of the self-dual Yang-Mills corresponding to the deformation of the topological A model by the operator $s \Phi_{3,3}$. In a realization á la Siegel $[74,75]$,

$$
S=\int d^{4} x \operatorname{Tr} F \wedge G
$$

the self-dual Yang-Mills is written in terms of a $U(N)$ adjoint valued selfdual 2-form $G$ and the curvature of a $U(N)$ connection, $F$. We can deform the action (9.4) by the term $\epsilon G \wedge G$, which (perturbatively) leads to the full Yang-Mills theory. It is natural to ask whether this deformation is dual to the deformation of the A model on $\mathbf{C P}^{3}$ by $s \Phi_{3,3}$. Notice, that bosonic Yang-Mills on $\mathbf{S}^{4}$ has partition function which depends on the radius of the 4-sphere, $R$, the coupling constant of Yang-Mills theory, $g_{Y M}^{2}$, and the rank $N$ of the gauge group. Due to the running of the coupling constant only one combination of $g_{Y M}^{2}$ and $R$ appears. It is not unreasonable to suppose that 
with a suitable choice of the parameter map (which should involve some kind of Fourier transform) we have

$$
Z_{\mathbf{C P}^{3}}^{\text {top }}\left(g_{s}, s^{2} e^{-t}\right) \leftrightarrow Z_{\mathbf{S}^{4}}^{Y M}\left(g_{Y M}^{2}, N\right)
$$

It would be very interesting to further develop and check this conjecture. If correct, it would allow one to place the appearance of the higher-dimensional twistor space $T(M)$ in the large $N$ limit of gauge theory on $M$ into the context of more familiar large $N$ dualities, e.g., the duality between ChernSimons theory on $S^{3}$ and topological strings on the 6-dimensional resolved conifold [73].

\section{Directions for future research}

In this paper, we have discussed the fact that many theories of gravity fall into the general class of "form gravity theories," and that they seem to be unified into a 7-dimensional theory of gravity, topological M-theory, related to $G_{2}$ holonomy metrics. We have seen in particular that this 7 dimensional theory contains the A and B model topological strings, which appear as conjugate degrees of freedom. We have also seen connections with 3-dimensional Chern-Simons gravity and a 4-dimensional form theory of gravity - the topological sector of loop quantum gravity.

Intriguing as this list is, we view this as only a modest beginning: the connections we have outlined raise many new questions which need to be answered. In order to understand better the non-perturbative aspects of the $\mathrm{A}$ and $\mathrm{B}$ models, and particularly their implementation in the context of topological M-theory, we need to understand better the relation between these models and M-theory. In particular, it seems natural to try to explain the S-duality relating the A and B models using the S-duality of Type IIB superstrings. This could be embedded into the present discussion if we include one more dimension and consider eight-dimensional manifolds of special holonomy. The natural candidate in that dimension are manifolds with $\operatorname{Spin}(7)$ holonomy. It seems that we also need to include this theory in our discussion of dualities to get a better handle on the S-duality of the A/B models.

Another natural question we have raised relates to the interpretation of the topological M-theory: does it indeed count domain walls? This is a very natural conjecture based on the links we found between form theories of gravity and the counting of black hole states. It would be important to develop this idea more thoroughly. 
Another question raised by our work is whether one can reformulate the full M-theory in terms of form theories of gravity. This may not be as implausible as it may sound at first sight. For example, we do know that $\mathcal{N}=2$ supergravity in 4 dimensions, which is a low energy limit of superstrings compactified on Calabi-Yau manifolds, has a simple low energy action: it is simply the covariantized volume form on (4|4) chiral superspace [76]. In fact, more is true: we could include the Calabi-Yau internal space as and write the leading term in the effective action as the volume element in dimension (10|4). The internal volume theory in this case would coincide with that of Hitchin. Indeed, this is related to the fact that topological string amplitudes compute F-terms in the corresponding supergravity theory. Given this link it is natural to speculate that the full M-theory does admit such a low energy formulation, which could be a basis of another way to quantize M-theory - rather in tune with the notion of quantum gravitational foam.

We have also discussed a speculation, motivated by topological M-theory, relating gauge theories on $M^{4}$ to topological strings on its twistor space. This connection, even though it needs to be stated more sharply, is rather gratifying, because it would give a holographic explanation of the fact that in the twistor correspondence a 4-dimensional theory gets related to a theory in higher dimensions. It would be very interesting to develop this conjectural relation; the potential rewards are clearly great, as a full understanding of the duality could lead to a large $N$ solution of non-supersymmetric Yang-Mills.

\section{Acknowledgments}

We are grateful to M. Atiyah, J. de Boer, R. Bryant, C. LeBrun, J. Louis, H. Ooguri, M. Roček, L. Smolin, C. Taubes, E. Verlinde, and S.-T. Yau for valuable discussions. We would like to thank the 2004 Simons Workshop on Mathematics and Physics and the Aspen Workshop "Strings, Branes and Superpotentials," which led to the development of many of the ideas in this paper. We also thank the organizers of the Strings 2004 conference in Paris for providing a stimulating environment where part of this work was done. S.G. and A.N. would like to thank Caltech Particle Theory Group, where part of this work was done, for kind hospitality. This work was conducted during the period S.G. served as a Clay Mathematics Institute Long-Term Prize Fellow. S.G. was also supported in part by RFBR grant 04-02-16880. The research of A.N. and C.V. was supported in part by NSF grants PHY-0244821 and DMS-0244464. The research of R.D. was partly supported by FOM and the NWO Spinoza premium. 


\section{Appendix A Hitchin's Hamiltonian flow and geometry of $\mathcal{N}=1$ string vacua}

The geometric structures which appear in the 7-dimensional topological gravity are reminiscent of the geometries that arise in $\mathcal{N}=1$ superstring compactifications. For example, 7 -manifolds with $G_{2}$ holonomy are classical solutions in 7-dimensional topological gravity and, on the other hand, are $\mathcal{N}=1$ vacua of $M$-theory. This relation can be extended to 6 -manifolds with $S U(3)$ structure which play an important role in understanding the space of string vacua with minimal $(\mathcal{N}=1)$ supersymmetry, and which we briefly review in this appendix; see [77-85] for more details.

Let $M$ be a 6-manifold with $S U(3)$ structure. Such $M$ are characterized by the existence of a globally defined, $S U(3)$ invariant spinor $\xi$, which is the analog of the covariantly constant spinor one has on a Calabi-Yau manifold. In general, instead of $\nabla \xi=0$ we have

$$
\nabla^{(T)} \xi=0
$$

where $\nabla^{(T)}$ is a connection twisted by torsion $T$. Roughly speaking, the intrinsic torsion $T$ represents the deviation from the Calabi-Yau condition. Its $S U(3)$ representation content involves five classes, usually denoted $\mathcal{W}_{i}$ $[86,87]$ :

$$
T \in \mathcal{W}_{1} \oplus \mathcal{W}_{2} \oplus \mathcal{W}_{3} \oplus \mathcal{W}_{4} \oplus \mathcal{W}_{5}
$$

In order to describe the geometric meaning of each of these components, it is convenient to introduce a 2 -form $k$ and a 3 -form $\Omega$,

$$
\begin{aligned}
k & =-i \xi^{\dagger} \Gamma_{m n} \Gamma_{7} \xi, \\
\Omega & =-i \xi^{\dagger} \Gamma_{m n p}\left(1+\Gamma_{7}\right) \xi,
\end{aligned}
$$

which satisfy

$$
k \wedge \Omega=0 .
$$

On a Calabi-Yau manifold, the 2-form $k$ would be the usual Kähler form, while $\Omega$ would be the holomorphic volume form. In particular, $M$ is a Calabi-Yau manifold if and only if $d k=0, d \Omega=0$. On a general manifold $M$ with $S U(3)$ structure, these equations are modified by the components of torsion,

$$
\begin{aligned}
& d k=-\frac{3}{2} \operatorname{Im}\left(W_{1} \bar{\Omega}\right)+W_{4} \wedge k+W_{3}, \\
& d \Omega=W_{1} k^{2}+W_{2} \wedge k+\bar{W}_{5} \wedge \Omega,
\end{aligned}
$$


where

$$
\begin{aligned}
& W_{1} \in \Omega^{0}(M), \\
& W_{2} \in \Omega^{2}(M), \\
& W_{3}=\bar{W}_{3} \in \Omega_{\text {prim }}^{2,1}(M) \oplus \Omega_{\text {prim }}^{1,2}(M), \\
& W_{4}=\bar{W}_{4} \in \Omega^{1}(M), \\
& W_{5} \in \Omega^{1,0}(M) .
\end{aligned}
$$

A particularly interesting class of manifolds with $S U(3)$ structure are the so-called half-flat manifolds. In superstring theory, they play an important role in constructing realistic vacua with minimal $(\mathcal{N}=1)$ supersymmetry, and can be viewed as mirrors of Calabi-Yau manifolds with (a particular kind of) NS-NS fluxes [77]. Since under mirror symmetry 3-forms are mapped into forms of even degree, on half-flat manifolds one might expect "NS-NS fluxes" represented by forms of even degree [88]. In fact, as we explain in a moment, on a half-flat manifold $M$ we have

$$
d(\operatorname{Im} \Omega) \sim F_{4}^{N S} .
$$

Half-flat manifolds are defined by requiring certain torsion components to vanish,

$$
\operatorname{Re} \mathcal{W}_{1}=\operatorname{Re} \mathcal{W}_{2}^{-}=\mathcal{W}_{4}=\mathcal{W}_{5}=0
$$

It is easy to see from equation (A.5) that this is equivalent to the conditions

$$
\begin{aligned}
& d(k \wedge k)=0, \\
& d(\operatorname{Re} \Omega)=0 .
\end{aligned}
$$

If as usual we define $\sigma=\frac{1}{2} k \wedge k$ and $\Omega=\rho+i \widehat{\rho}$, we can write these equations in the familiar form

$$
\begin{aligned}
& d \rho=0 \\
& d \sigma=0
\end{aligned}
$$

with an additional constraint $\rho \wedge k=0$. This is precisely the structure induced on a generic 6 -dimensional hypersurface inside a $G_{2}$ manifold, where $\rho$ is the pull-back of the associative 3 -form $\Phi$ and $\sigma$ is the pull-back of the coassociative 4-form $* \Phi$. In particular, using Hitchin's Hamiltonian flow which we reviewed in Section 4.5, a half-flat $S U(3)$ structure on $M$ can always be thickened into a $G_{2}$ holonomy metric on $X=M \times(a, b)$. 
So the phase space underlying Hitchin's Hamiltonian flow consists precisely of the half-flat manifolds which appear in $\mathcal{N}=1$ string compactifications with fluxes and/or torsion,

$$
\mathcal{P}_{\text {Hitchin }}=\left\{M_{\text {half-flat }}^{6}\right\} .
$$

Moreover, the ground states are related to stationary solutions of Hitchin's flow equations, namely Calabi-Yau manifolds,

$$
\text { |vac }\rangle \quad \Longleftrightarrow \quad M^{6}=\text { Calabi-Yau. }
$$

It is tempting to speculate that all $\mathcal{N}=1$ string vacua can be realized in topological M-theory.

\section{References}

[1] A. Neitzke and C. Vafa, $N=2$ strings and the twistorial Calabi-Yau, hep-th/0402128.

[2] N. Nekrasov, H. Ooguri and C. Vafa, S-duality and topological strings, hep-th/0403167.

[3] A. Okounkov, N. Reshetikhin and C. Vafa, Quantum Calabi-Yau and classical crystals, hep-th/0309208.

[4] A. Iqbal, N. Nekrasov, A. Okounkov and C. Vafa, Quantum foam and topological strings, hep-th/0312022.

[5] H. Ooguri, A. Strominger and C. Vafa, Black hole attractors and the topological string, hep-th/0405146.

[6] C. Vafa, Two dimensional Yang-Mills, black holes and topological strings, hepth/0406058.

[7] M. Bershadsky, S. Cecotti, H. Ooguri and C. Vafa, Kodaira-Spencer theory of gravity and exact results for quantum string amplitudes, Commun. Math. Phys. 165 (1994), 311.

[8] E. Witten, Quantum background independence in string theory, hep-th/9306122.

[9] R. Dijkgraaf, E. Verlinde and M. Vonk, On the partition sum of the NS five-brane, hep-th/0205281.

[10] N. Hitchin, Stable forms and special metrics, math.DG/0107101.

[11] J.A. Harvey and G.W. Moore, Superpotentials and membrane instantons, hepth/9907026.

[12] M. Aganagic, A. Klemm and C. Vafa, Disk instantons, mirror symmetry and the duality web, Z. Naturforsch. A57 (2002), 1-28 hep-th/0105045. 
[13] M. Aganagic and C. Vafa, G(2) manifolds, mirror symmetry and geometric engineering, hep-th/0110171.

[14] M. Bershadsky, S. Cecotti, H. Ooguri and C. Vafa, Holomorphic anomalies in topological field theories, Nucl. Phys. B405 (1993), 279.

[15] H. Ooguri and C. Vafa, Geometry of $N=2$ strings, Nucl. Phys. B361 (1991), 469-518.

[16] M. Aganagic, R. Dijkgraaf, A. Klemm, M. Marino and C. Vafa, Topological strings and integrable hierarchies, hep-th/0312085.

[17] M. Aganagic, A. Klemm, M. Marino and C. Vafa, The topological vertex, hepth/ 0305132.

[18] A. Achucarro and P.K. Townsend, A Chern-Simons action for 3dimensional anti-De Sitter supergravity theories, Phys. Lett. B180 (1986), 89.

[19] E. Witten, (2+1)-dimensional gravity as an exactly soluble system, Nucl. Phys. B311 (1988), 46.

[20] V.G. Turaev and O.Yu. Viro, State-sum invariants of 3-manifolds and quantum 6j-symbols, Topology 31 (1992), 865.

[21] V.G. Turaev, C. R. Acad. Sci. Paris, t. 313, Série I 395 (1991); “Topology of Shadow," preprint (1991).

[22] K. Walker, On Witten's 3-Manifold invariants, unpublished.

[23] E. Witten, Quantum field theory and the jones polynomial, Commun. Math. Phys. 121 (1989), 351.

[24] N.Y. Reshetikhin and V.G. Turaev, Invariants of 3-manifolds via link polynomials and quantum groups, Invent. Math. 103, (1991) 547.

[25] S. Gukov, 3-dimensional quantum gravity, Chern-Simons theory, and the Apolynomial, hep-th/0306165.

[26] M. Plebanski, On the separation of Einsteinian substructures, J. Math. Phys. 18 (1977), 2511.

[27] G. Horowitz, Exactly soluble diffeomorphism invariant theories, Commun. Math. Phys. 125 (1989), 417.

[28] C. Torre, Perturbations of gravitational instantons, Phys. Rev. D41 (1990), 3620 .

[29] C. Torre, A topological field theory of gravitational instantons, Phys. Lett. B252 (1990), 242-246.

[30] M. Abe, A. Nakamichi and T. Ueno, Moduli space of topological two form gravity, Mod. Phys. Lett. A9 (1994), 895-901; hep-th/9306130.

[31] H.Y. Lee, A. Nakamichi and T. Ueno, Topological two form gravity in four dimensions, Phys. Rev. D47 (1993), 1563-1568; hep-th/9205066. 
[32] A. Nakamichi, Wave function of the universe in topological and in Einstein two form gravity, hep-th/9303135.

[33] M. Abe, A. Nakamichi and T. Ueno, Gravitational instantons and moduli spaces in topological two form gravity, Phys. Rev. D50 (1994), 7323; hep-th/9408178.

[34] L. Smolin, An invitation to loop quantum gravity, hep-th/0408048.

[35] W. Israel, Differential Forms in General Relativity, Comm. Dublin Inst. Adv. Stud. Series A 26 (Dublin).

[36] R. Capovilla, T. Jacobson, J. Dell and L. Mason, Self-dual 2-forms and gravity, Class. Quant. Grav. 8 (1991), 41-57.

[37] H. Urbantke, J. Math. Phys. 25 (1983), 2321.

[38] N.J. Hitchin, Kählerian twistor spaces, Proc. London Math. Soc. 43(1) (1981), 133.

[39] M. Bershadsky and V. Sadov, Theory of Kahler gravity, Int. J. Mod. Phys. A 11 (1996), 4689; hep-th/9410011.

[40] N. Berkovits and E. Witten, Conformal supergravity in twistor-string theory, hepth/0406051.

[41] E. Witten, Chern-Simons gauge theory as a string theory, Prog. Math. 133 (1995), 637; hep-th/9207094.

[42] N. Hitchin, The geometry of 3-forms in six and seven dimensions, mathDG/0010054.

[43] S. Gukov, S.T. Yau and E. Zaslow, Duality and fibrations on G(2) manifolds, hep-th/0203217.

[44] Z.W. Chong, M. Cvetic, G.W. Gibbons, H. Lu, C.N. Pope and P. Wagner, General metrics of G(2) holonomy and contraction limits, Nucl. Phys. B638 (2002), 459; hep-th/0204064.

[45] M. Cvetic, G.W. Gibbons, H. Lu and C.N. Pope, Bianchi IX self-dual Einstein metrics and singular G(2) manifolds, Class. Quant. Grav. 20 (2003), 4239-4268; hepth/0206151.

[46] D. Maulik, N. Nekrasov, A. Okounkov and R. Pandharipande, GromovWitten theory and Donaldson-Thomas theory, I-II, math.AG/0312059; math.AG/0406092.

[47] L. Baulieu and I.M. Singer, Topological Yang-Mills symmetry, Nucl. Phys. Proc. Suppl. 5B (1988), 12.

[48] L. Baulieu, H. Kanno and I.M. Singer, Special quantum field theories in eight and other dimensions, Commun. Math. Phys. 194 (1998), 149; hep-th/9704167.

[49] C. Hofman and J.S. Park, Cohomological Yang-Mills theories on Kähler 3-folds, Nucl. Phys. B600 (2001), 133; hep-th/0010103. 
[50] S.K. Donaldson and R.P. Thomas, Gauge theory in higher dimensions, in The Geometric Universe; Science, Geometry, And The Work Of Roger Penrose, Oxford University Press, 1998.

[51] B.S. Acharya, M. O'Loughlin and B. Spence, Higher-dimensional analogues of Donaldson-Witten theory, Nucl. Phys. B503 (1997), 657; hep-th/9705138.

[52] M. Blau and G. Thompson, Euclidean SYM theories by time reduction and special holonomy manifolds, Phys. Lett. B415 (1997), 242; hepth/9706225.

[53] C. Vafa and E. Witten, A strong coupling test of $S$ duality, Nucl. Phys. B431 (1994), 3; hep-th/9408074.

[54] A.A. Gerasimov and S.L. Shatashvili, Towards integrability of topological strings. I: 3-forms on Calabi-Yau manifolds, hep-th/0409238.

[55] R. Bryant and S. Salamon, On the construction of some complete metrics with exceptional holonomy, Duke Math. J. 58 (1989), 829.

[56] G.W. Gibbons, D.N. Page and C.N. Pope, Einstein Metrics on $S^{3}, R^{3}$, and $R^{4}$ Bundles, Commun. Math. Phys. 127 (1990), 529-553.

[57] A. Kapustin, Gauge theory, topological strings, and S-duality, JHEP 0409 (2004), 034; hep-th/0404041.

[58] A. Strominger, Macroscopic entropy of $N=2$ extremal black holes, Phys. Lett. B383 (1996), 39-43; hep-th/9602111.

[59] S. Ferrara, R. Kallosh and A. Strominger, $N=2$ extremal black holes, Phys. Rev. D52 (1995), 5412-5416; hep-th/9508072.

[60] S. Ferrara and R. Kallosh, Supersymmetry and attractors, Phys. Rev. D 54 (1996), 1514; hep-th/9602136.

[61] S. Ferrara and R. Kallosh, Universality of supersymmetric attractors, Phys. Rev. D 54 (1996), 1525; hep-th/9603090.

[62] A. Chou, R. Kallosh, J. Ramfeld, S. Rey, M. Shmakova and W.K. Wong, Critical points and phase transitions in 5d compactifications of M-theory, Nucl. Phys. B508 (1997), 147-180; hep-th/9704142.

[63] R. Gopakumar and C. Vafa, M-theory and topological strings. II, hepth/9812127.

[64] M.F. Atiyah, N. Hitchin and I.M. Singer, Self-duality in 4-dimensional Riemannian geometry, Proc. Roy. Soc. London Ser. A 362 (1978), 425461.

[65] R. Penrose, Nonlinear gravitons and curved twistor theory, Gen. Rel. Grav. 7 (1976), 31-52.

[66] C. LeBrun, Twistors for tourists: a pocket guide for algebraic geometers, Proc. Sympos. Pure Math., 62, Part 2, (1997), 361. 
[67] E. Witten, Perturbative gauge theory as a string theory in twistor space, hepth/0312171.

[68] A. Givental and B. Kim, Quantum cohomology of flag manifolds and Toda lattices, Commun. Math. Phys. 168 (1995), 609; hep-th/9312096.

[69] M. Kontsevich and Yu. Manin, Gromov-Witten classes, quantum cohomology, and enumerative geometry, Commun. Math. Phys. 164 (1994), 525; hep-th/9402147.

[70] A. Bayer and Yu. Manin, (Semi) simple exercises in quantum cohomology, math- AG/0103164.

[71] T. Eguchi, K. Hori and C.-S. Xiong, Gravitational quantum cohomology, Int. J. Mod. Phys. A12 (1997), 1743; hep-th/9605225.

[72] P. Horava and E. Witten, Heterotic and type I string dynamics from eleven dimensions, Nucl. Phys. B460 (1996), 506-524; hep-th/9510209.

[73] R. Gopakumar and C. Vafa, Topological gravity as large $N$ topological gauge theory, Adv. Theor. Math. Phys. 2 (1998), 413; hep-th/9802016. On the gauge theory/geometry correspondence, Adv. Theor. Math. Phys. 3, 1415 (1999), hepth/9811131.

[74] W. Siegel, $N=2, N=4$ string theory is selfdual $N=4$ Yang-Mills theory, Phys. Rev. D46 (1992), 3235; "Selfdual N=8 supergravity as closed $N=2(N=4)$ strings," Phys. Rev. D47, 2504 (1993), hepth/9207043.

[75] G. Chalmers and W. Siegel, The self-dual sector of QCD amplitudes, Phys. Rev. D54 (1996), 7628; hep-th/9606061.

[76] E. Sokatchev, A superspace action for $N=2$ supergravity, Phys. Lett. B100 (1981), 466.

[77] S. Gurrieri, J. Louis, A. Micu and D. Waldram, Mirror symmetry in generalized Calabi-Yau compactifications, Nucl. Phys. B654 (2003), 61; hep-th/0211102.

[78] G.L. Cardoso, G. Curio, G. Dall'Agata, D. Lust, P. Manousselis and G. Zoupanos, Non-Kähler string backgrounds and their five torsion classes, Nucl. Phys. B652 (2003), 5; hep-th/0211118.

[79] S. Kachru, M.B. Schulz, P.K. Tripathy and S.P. Trivedi, New supersymmetric string compactifications, JHEP 0303 (2003), 061; hepth/0211182.

[80] S. Gurrieri and A. Micu, Type IIB theory on half-flat manifolds, Class. Quant. Grav. 20 (2003), 2181; hep-th/0212278.

[81] P. Kaste, R. Minasian, M. Petrini and A. Tomasiello, Nontrivial RR 2form field strength and SU(3)-structure, Fortsch. Phys. 51 (2003), 764; hep-th/0301063. 
[82] K. Becker, M. Becker, K. Dasgupta and P.S. Green, Compactifications of heterotic theory on non-Kähler complex manifolds. I, JHEP 0304 (2003), 007; hep-th/0301161.

[83] J.P. Gauntlett, D. Martelli and D. Waldram, Superstrings with intrinsic torsion, Phys. Rev. D69 (2004), 086002; hep-th/0302158.

[84] M. Grana, R. Minasian, M. Petrini and A. Tomasiello, Supersymmetric backgrounds from generalized Calabi-Yau manifolds, JHEP 0408 (2004), 046; hep-th/0406137.

[85] S. Gurrieri, A. Lukas and A. Micu, Heterotic on half-flat, hepth/0408121.

[86] S. Salamon, Almost Parallel Structures, Contemp. Math. 288 (2001), 162; math-DG/0107146.

[87] S. Chiossi and S. Salamon, The intrinsic torsion of SU(3) and G2 structures, math.DG/0202282.

[88] C. Vafa, Superstrings and topological strings at large N, J. Math. Phys. 42 (2001), 2798; hep-th/0008142. 
